A. Fiolitakis, C. M. Arndt, "Transported PDF simulation of auto-ignition of a turbulent methane jet in a hot, vitiated coflow", Combustion Theory and Modelling, 24 (2020) 326-361

The Version of Record of this manuscript has been published and is available in Combustion Theory and Modelling, published online: 23 October 2019 http://www.tandfonline.com/10.1080/13647830.2019.1682197 


\title{
Transported PDF simulation of auto-ignition of a turbulent methane jet in a hot, vitiated coflow
}

\author{
A. Fiolitakis ${ }^{\mathrm{a}}$ and C. M. Arndt ${ }^{\mathrm{a}}$ \\ ${ }^{a}$ German Aerospace Center, Pfaffenwaldring 38-40, 70569 Stuttgart, Germany
}

\begin{abstract}
ARTICLE HISTORY
Compiled October 15, 2019

ABSTRACT

In the current work, the auto-ignition of a turbulent round methane jet is studied numerically by means of a transported probability density function (PDF) method. The methane jet is issued into a hot, vitiated coflow, where it ignites to form a steady lifted flame. For this flame, experimental data of hydroxyl, temperature and mixture fraction are provided in the area where the fuel auto-ignites. To model this experiment, the transport equation for the thermochemical PDF is solved using a hybrid finite volume / Lagrangian Monte-Carlo method. Turbulence is modeled using the $k-\varepsilon$ turbulence model including a jet-correction. Computational results are compared to experimental data in terms of mean quantities, variances and liftoff height. Moreover, the structure of the one-point, one-time marginal PDF of temperature is analyzed and compared to experimental data which are provided in this work. It is found that the transported PDF method in conjunction with the $k-\varepsilon$ model is capable of reproducing these statistical data very well. In particular the effect of ignition on the marginal PDF of temperature can be well reproduced with this approach. To further analyze the relevant processes in the evolution of the temperature PDF, a statistically homogeneous system is studied both numerically and analytically.
\end{abstract}

\section{KEYWORDS}

Transported PDF; Lagrangian Monte-Carlo method; auto-ignition; flame lift-off; temperature PDF

\section{Introduction}

Auto-ignition in turbulent flows is of great technical importance and has therefore been studied extensively in the past. Foremost, controlling auto-ignition is important for the safety of technical systems. Auto-ignition is also a key element in many technical combustion processes like diesel engines (see e.g. [51]). Also in stationary gas turbines, combustion concepts are found where staged combustion requires the control of auto-ignition (cf. [58]).

For these reasons, modeling of auto-ignition is of great interest. To model the behavior of non-premixed systems undergoing auto-ignition, a series of combustion models exists (a comprehensive overview on this topic is provided by Mastorakos [65]). One widely used approach is the Conditional Moment Closure (CMC), where

CONTACT Andreas Fiolitakis. Email: andreas.fiolitakis@dlr.de. Phone: +497116862352

CONTACT Christoph Michael Arndt. Email: christoph.arndt@dlr.de. Phone: +497116862445 
transport equations for the conditional mean of a scalar variable are solved. The conditional expectation is usually formed with respect to mixture fraction [54]. One distinctive advantage of the CMC method is the explicit presence of the conditional scalar dissipation rate in the model equations [65]. The statistical distribution of scalar dissipation rate at a given mixture fraction, which is an important aspect for modeling auto-ignition, is thus included in CMC [65]. This approach has been widely used to investigate auto-ignition in turbulent lifted flames [21, 23, 71-73, 77, 88, 90, 91, 95, 96] or in turbulent jets $[18,53]$. It has also been applied for the simulation of auto-ignition in sprays $[16,17,104]$ and stratified mixtures [12].

In addition to the CMC model, various flamelet models have been applied in the past for the simulation of auto-ignition. The underlying assumption of the flamelet model is that a turbulent flame may be viewed as an ensemble of "reactive-diffusive layers" which moves randomly in the turbulent flow [78]. The advantage of this model approach is that, as in the $\mathrm{CMC}$ approach, the effect of scalar dissipation appears inherently in the model equations. In contrast to CMC, the effect of fluctuations in scalar dissipation rate is usually not included [65]. Simulations of lifted flames using flamelet models are found in [10, 39, 43, 70]. Auto-ignition in a MILD combustor is addressed in the work of Ihme and See [44], and auto-ignition of gaseous jets is investigated by Kim et al. [52]. A study of auto-ignition of hydrogen-air mixtures in mixture fraction space with unsteady flamelets is given in [89]. And finally, auto-ignition of spray is investigated in [24, 25, 29, 43, 56, 60, 61, 74, 94, 105].

Related approaches for auto-ignition modeling are the flamelet-generated-manifolds (FGM) $[1,2,13,14,29,36,42]$, the flame prolongation of intrinsic low-dimensional manifolds (FPI) [26], and the approximated diffusion flame-presumed conditional moment (ADF-PCM) method [66].

In addition to these approaches, Chen et al. [20] used a method based on premixed flamelets for the simulation of lifted methane jet flames. The model used in [20] incorporates the effect of non-premixed combustion as well as the statistical correlation between reaction progress variable and mixture fraction. This statistical correlation is accomplished via a copula in the presumed joint probability density function of mixture fraction and reaction progress variable. Usually, these quantities are assumed to be statistically independent.

The present paper utilizes the transported probability density function (TPDF) method which is described in Sec. 3. Compared to other modeling approaches, this method offers the distinctive advantage that the chemical source term appears as an algebraically closed expression, i.e. no further modeling assumptions are required for its treatment. One disadvantage is that micro mixing requires modeling. Many earlier works utilize various TPDF and filtered density function (FDF) formulations for the simulation of auto-ignition. Investigations of auto-ignition in different systems are presented in $[15,19,34,35,38,40,41,46,49,50,59,64,67,86,101,106]$. However, the lifted methane jet flame, which is being modeled in this work, has not been studied yet by means of the TPDF method (first results of large-eddy simulation of the burner are presented in [84]). Details on the experiment conducted by Arndt et al. [3, 5] are given in Sec. 2. Results for various statistical quantities are given in Sec. 4. A novel aspect in this work is the experimental as well as the numerical investigation of the marginal one-point, one-time probability density function (PDF) of temperature, which (to our knowledge) has not been studied yet in lifted, auto-ignition stabilized flames. Earlier work on similar flames (see e.g. $[15,19,41,59,64,67,101]$ ) is focused on a qualitative 
investigation of temperature distribution or provides PDFs without any reference to experimental data [49]. Only in the work of Gkagkas and Lindstedt [34, 35] are PDFs of temperature directly compared to experimental data. These PDFs are, however, conditioned on mixture fraction and formulated for samples across the entire flame. A quantification of the one-point, one-time PDF of temperature has not been attempted yet. This is, however, an important parameter for auto-ignition as the occurrence of high temperatures may trigger ignition, provided that favorable conditions exist in terms of mixing and scalar dissipation rate [65]. Hence, special emphasis is given here to the investigation of the marginal PDF of temperature.

\section{Testcase}

\subsection{Description of Experimental Setup}

The auto-ignition experiment, which is the subject of the investigations in the present work, has been conducted by Arndt et al. [7, 9] on the "DLR (Deutsches Zentrum für Luft- und Raumfahrt (Germany Aerospace Center)) JHC (jet in hot coflow)" burner $[4,6]$. In this experiment, the auto-ignition of a methane jet in a coflow of hot, vitiated air is investigated. Figure 1 illustrates the DLR-JHC burner. Through a nozzle of $1.5 \mathrm{~mm}$ diameter, which is placed in the center of a square combustion chamber, a jet of methane is ejected into a co-axial coflow of hot, vitiated air. The coflow consists of the oxygen-containing exhaust gas of a lean-premixed hydrogen air flame (equivalence ratio of 0.466$)$, which is stabilized on a matrix burner $(75 \mathrm{~mm} \times 75 \mathrm{~mm})$ at the bottom of the combustion chamber. The composition of burnt gases is given in [9] and summarized in Tab. 1. The coflow temperature is $1490 \mathrm{~K}$ and its velocity is $4.1 \mathrm{~m} / \mathrm{s}$. The chamber is equipped with four quartz glass windows at its sides such that access is provided for optical measuring techniques and entrainment of surrounding air is avoided. The chamber is open at its top so that all measurements are conducted at atmospheric pressure.

Different processes, which include both transient ignition processes as well as stable

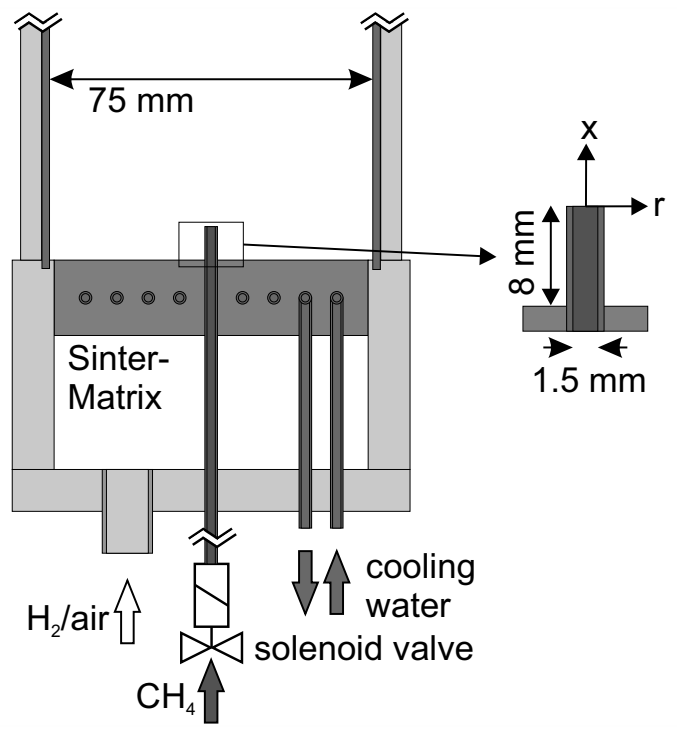

Figure 1. Sketch of DLR-JHC burner. 
Table 1. Composition of coflow in mole fractions [9].

\begin{tabular}{l|cccc} 
species & $\mathrm{O}_{2}$ & $\mathrm{H}_{2} \mathrm{O}$ & $\mathrm{N}_{2}$ & $\mathrm{OH}$ \\
\hline mole fraction & 0.1021 & 0.1782 & 0.7196 & 0.00163
\end{tabular}

lifted flames, have been studied experimentally by Arndt et al. [7, 9]. The present work aims at investigating a steady, lifted flame. The operating conditions of this flame are described in detail in [7]. With a methane temperature of $290 \mathrm{~K}$ (in the simulation) and a methane mass flow of $0.2067 \mathrm{~g} / \mathrm{s}$, a diameter-based Reynolds number of approximately 16,000 is found for the methane jet.

\subsection{Measurements of Mixture Fraction, Temperature and $\mathrm{OH}$ Concentration}

Details of the measurement procedure and data processing for the mixture fraction and temperature $[7,75]$ and $\mathrm{OH}$ concentration measurements $[5,8]$ can be found in literature, and only the key parameters are described here. For the mixture fraction and temperature measurements, planar laser Rayleigh scattering is performed using the High Energy Pulse Burst Laser System (HEPBLS) at the Ohio State University $[33,76]$. The laser system produces bursts of 230 consecutive laser pulses at a pulse repetition rate of $10 \mathrm{kHz}$ with a pulse energy of $>1 \mathrm{~J}$ at $532 \mathrm{~nm}$, and a pulse duration of $25 \mathrm{~ns}$. Since the laser beam has a diameter of approximately $15 \mathrm{~mm}$ in the test section, no beam expansion is employed and the laser-sheet is formed by focusing the beam into the test section with a single plano-convex cylindrical lens $(f=750 \mathrm{~mm})$, resulting in a laser-sheet thickness in the test section of $<300 \mu \mathrm{m}$. Rayleigh scattering from the test section is collected using a high speed CMOS camera (Phantom v711) in combination with an achromat lens $(D=100 \mathrm{~mm}, f=240 \mathrm{~mm})$ and a fast camera lens $(f=85 \mathrm{~mm}, f / 1.4)$. Adjacent to the test section, a reference air stream with known temperature is placed. The reference air stream is used to perform singleshot laser intensity and laser beam profile corrections, using an additional high speed CMOS camera (Phantom v711). After darkfield and flatfield correction as well as spatial calibration, the mixture fraction and temperature fields are calculated using a multi-step process, which is described in detail in [75]. The resulting measurement uncertainty is $<2 \%$, including uncertainties of the mass flow controllers and of the Rayleigh measurement system.

The $\mathrm{OH}$ concentration is measured using high-speed $\mathrm{OH}$ planar laser-induced fluorescence (PLIF). A frequency doubled dye laser (Sirah Credo, pulse repetition rate $10 \mathrm{kHz}$, pulse energy $0.1 \mathrm{~mJ}$ at $283.2 \mathrm{~nm}$ ) is pumped using a frequency doubled diodepumped solid-state (DPSS) laser (EdgeWave InnoSlab IS8II-E) and is tuned to match the Q1(7) transition in the $\mathrm{A}-\mathrm{X}(1,0)$ band of the $\mathrm{OH}$ radical. The laser beam is expanded into a light sheet using a cylindrical Galilean telescope and focused into the test section using a third cylindrical lens, resulting in a $40 \mathrm{~mm}$ high laser sheet with a $400 \mu \mathrm{m}$ beam waist in the test section. The fluorescence signal in the $(0,0)$ and $(1,1)$ band is collected using an intensified high-speed CMOS camera (LaVision HSS8 with LaVision HS-IRO), equipped with a fast UV lens (Cerco, $f=45 \mathrm{~mm}, f / 1.8$ ) and a high-transmission bandpass filter (transmission $>80 \%$ at $310 \mathrm{~nm}$ ). Calibration and quantification of the measurement signal is performed based on the relation between the $\mathrm{OH}$ PLIF signal and the $\mathrm{OH}$ concentration in the coflow, using a section of the coflow with a known equilibrium $\mathrm{OH}$ concentration. For quantifying the signal in re- 
acting regions, different additional quantities, such as temperature and composition dependent quenching and the temperature dependence of the Boltzmann fraction in the ground state, have to be considered. Details on the data processing and error estimation can be found in $[5,8]$. The total measurement uncertainty for the mean $\mathrm{OH}$ concentration is on the order of $25 \%$ for the coflow region and between $+60 /-25 \%$ in stoichiometric, reacting regions.

\section{Model Description}

\subsection{PDF Model Equation}

To model auto-ignition in a turbulent flow, the TPDF method is used in this work, where a transport equation for the one-point, one-time joint PDF of the thermochemical quantities is solved. Since incompressible flows are considered in this work (i.e. the thermodynamic pressure is assumed to be constant, cf. [81]), the thermodynamic state is completely described by the specific enthalpy $h$ and the mass fractions $Y_{i}$ of the linearly independent species. For these quantities the transport equation of the joint one-point one-time mass density function (MDF) reads [81] (Einstein notation)

$$
\begin{aligned}
\frac{\partial \mathscr{F}_{\phi}}{\partial t}+\frac{\partial}{\partial x_{\alpha}}\left(\left\langle u_{\alpha} \mid \boldsymbol{\psi}\right\rangle \mathscr{F}_{\phi}\right) & =\frac{\partial}{\partial \hat{h}}\left(\mathscr{F}_{\phi}\left\langle\frac{1}{\rho} \frac{\partial q_{\alpha}}{\partial x_{\alpha}} \mid \boldsymbol{\psi}\right\rangle\right) \\
+ & \frac{\partial}{\partial \hat{Y}_{i}}\left(\mathscr{F}_{\phi}\left\langle\frac{1}{\rho} \frac{\partial j_{i \alpha}}{\partial x_{\alpha}} \mid \boldsymbol{\psi}\right\rangle\right)-\frac{\partial}{\partial \hat{Y}_{i}}\left(\mathscr{F}_{\phi} \frac{\dot{m}_{i}}{\rho}\right),
\end{aligned}
$$

where $\mathscr{F}_{\phi}$ is the MDF, i.e. the density weighted probability density function. In Eq. (1) $t$ represents time, $x_{\alpha}$ the spatial coordinate $(\alpha=1,2,3), u_{\alpha}$ the flow velocity, $\boldsymbol{\psi}=$ $\left(\hat{h}, \hat{Y}_{i}\right)^{T}\left(i=1, \ldots, N_{s}-1\right.$, where $N_{s}$ denotes the number of species) the sample space vector of the random vector $\boldsymbol{\phi}=\left(h, Y_{i}\right)^{T}, \rho$ the density, $q_{\alpha}$ the diffusive heat flux, $j_{i \alpha}$ the diffusive mass flux, and $\dot{m}_{i}$ the chemical source term. The operator $\langle\bullet \mid \boldsymbol{\psi}\rangle$ in Eq. (1) denotes the conditional expectation of a random quantity with respect to the sample space vector $\boldsymbol{\psi}$. Terms involving this conditional expectation are unclosed and require modeling. The second term on the left-hand-side of Eq. (1), the conditional spatial transport $\left\langle u_{\alpha} \mid \psi\right\rangle \mathscr{F}_{\phi}$, is modeled by decomposing the velocity $u_{\alpha}$ into it is Favre average $\tilde{u}_{\alpha}$ and Favre fluctuation $u_{\alpha}^{\prime \prime}$ yielding

$$
\left\langle u_{\alpha} \mid \boldsymbol{\psi}\right\rangle \mathscr{F}_{\phi}=\tilde{u}_{\alpha} \mathscr{F}_{\phi}+\left\langle u_{\alpha}^{\prime \prime} \mid \boldsymbol{\psi}\right\rangle \mathscr{F}_{\phi}
$$

The turbulent flux of the MDF is then finally closed by using a gradient diffusion model of Pope [80], i.e.

$$
\left\langle u_{\alpha}^{\prime \prime} \mid \psi\right\rangle \mathscr{F}_{\phi}=-\langle\rho\rangle D_{T} \frac{\partial}{\partial x_{\alpha}}\left(\frac{\mathscr{F}_{\phi}}{\langle\rho\rangle}\right)
$$

where $D_{T}$ denotes the turbulent diffusivity and the operator $\langle\bullet\rangle$ expectations.

In order to model the conditional expectation of the divergence of the diffusive fluxes in Eq. (1), the "interaction by exchange with the mean" (IEM) model [100] is used, 
yielding (Einstein notation)

$$
\begin{aligned}
& \left\langle\frac{1}{\rho} \frac{\partial j_{i \alpha}}{\partial x_{\alpha}} \mid \psi\right\rangle=\frac{1}{2} \frac{C_{\phi}}{\tau_{t}}\left(\hat{Y}_{i}-\tilde{Y}_{i}\right), \\
& \left\langle\frac{1}{\rho} \frac{\partial q_{\alpha}}{\partial x_{\alpha}} \mid \psi\right\rangle=\frac{1}{2} \frac{C_{\phi}}{\tau_{t}}(\hat{h}-\tilde{h}),
\end{aligned}
$$

where $\tau_{t}$ denotes the turbulence integral time scale and $C_{\phi}$ the mechanical-to-scalar timescale ratio (which equals 2). The operator $\approx$ represents Favre averages of the variables.

With these model approaches, the closed MDF transport equation reads (Einstein notation)

$$
\begin{aligned}
& \frac{\partial \mathscr{F}_{\phi}}{\partial t}+\frac{\partial}{\partial x_{\alpha}}\left(\left(\tilde{u}_{\alpha}+\frac{1}{\langle\rho\rangle} \frac{\partial\left(\langle\rho\rangle D_{T}\right)}{\partial x_{\alpha}}\right) \mathscr{F}_{\phi}\right)-\frac{\partial^{2}}{\partial x_{\alpha} \partial x_{\alpha}}\left(D_{T} \mathscr{F}_{\phi}\right) \\
& -\frac{\partial}{\partial \hat{h}}\left(\frac{1}{2} \frac{C_{\phi}}{\tau_{t}}(\hat{h}-\tilde{h}) \mathscr{F}_{\phi}\right)+\frac{\partial}{\partial \hat{Y}_{i}}\left(\left(\frac{\dot{m}_{i}}{\rho}-\frac{1}{2} \frac{C_{\phi}}{\tau_{t}}\left(\hat{Y}_{i}-\tilde{Y}_{i}\right)\right) \mathscr{F}_{\phi}\right)=0 .
\end{aligned}
$$

An efficient solution method for this equation is outlined in the following subsections.

In order to understand the evolution of the marginal PDF of temperature, it is in addition necessary to derive its transport equation from Eq. (6). To this end, Eq. (6) is transformed in thermochemical space from $\hat{h}-\hat{Y}_{i}$ coordinates to $\hat{T}-\hat{Y}_{i}$ coordinates, where $\hat{T}$ denotes the sample space variable of temperature. Subsequently, the joint MDF of enthalpy and composition $\mathscr{F}_{h, Y_{i}}$ is substituted by the joint MDF of temperature and composition $\mathscr{F}_{T, Y_{i}}$. Both MDFs are related to each other via [81]

$$
\mathscr{F}_{T, Y_{i}}\left(\hat{T}, \hat{Y}_{i}\right)=\mathscr{F}_{h, Y_{i}}\left(\hat{h}, \hat{Y}_{i}\right) \operatorname{det}\left(\frac{\partial\left(\hat{h}, \hat{Y}_{i}\right)}{\partial\left(\hat{T}, \hat{Y}_{i}\right)}\left(\hat{T}, \hat{Y}_{i}\right)\right),
$$

where $\partial\left(\hat{h}, \hat{Y}_{i}\right) / \partial\left(\hat{T}, \hat{Y}_{i}\right)$ denotes the Jacobian matrix of the transformation and det the determinant. To obtain a transport equation for the marginal PDF of temperature $P_{T}(\hat{T})$, the relation

$$
\mathscr{F}_{T, Y_{i}}\left(\hat{T}, \hat{Y}_{i}\right)=\rho P_{T}(\hat{T}) P_{Y_{i} \mid T}\left(\hat{Y}_{i} \mid \hat{T}\right)
$$

is used, with $P_{Y_{i} \mid T}\left(\hat{Y}_{i} \mid \hat{T}\right)$ being the conditional PDF of composition at given $\hat{T}$. The resulting equation is then integrated over composition space in order to obtain the transport equation for the marginal MDF of temperature $\mathscr{F}_{T}$, i.e. (Einstein notation)

$$
\begin{aligned}
& \frac{\partial \mathscr{F}_{T}}{\partial t}+\frac{\partial}{\partial x_{\alpha}}\left(\left(\tilde{u}_{\alpha}+\frac{1}{\langle\rho\rangle} \frac{\partial\left(\langle\rho\rangle D_{T}\right)}{\partial x_{\alpha}}\right) \mathscr{F}_{T}\right)-\frac{\partial^{2}}{\partial x_{\alpha} \partial x_{\alpha}}\left(D_{T} \mathscr{F}_{T}\right) \\
& +\frac{\partial}{\partial \hat{T}}\left(\left(\frac{1}{2} \frac{C_{\phi}}{\tau_{t}}\left\langle\frac{\tilde{h}-h_{i} \tilde{Y}_{i}}{c_{p}} \rho \mid \hat{T}\right\rangle+\left\langle-\frac{\dot{m}_{i} h_{i}}{c_{p}} \mid \hat{T}\right\rangle\right) \frac{\mathscr{F}_{T}}{\langle\rho \mid \hat{T}\rangle}\right)=0
\end{aligned}
$$


where the MDF is defined as the product of the marginal PDF of temperature and the conditional expectation of density, i.e.

$$
\mathscr{F}_{T}=\langle\rho \mid \hat{T}\rangle P_{T}
$$

In Eq. (9), $c_{p}$ denotes the specific heat capacity of the mixture and $h_{i}$ the specific enthalpy of a species. The first term under the temperature derivative of Eq. (9) describes the effect of micro mixing on the temperature MDF. It simplifies to (Einstein notation)

$$
\left\langle\frac{1}{2} \frac{C_{\phi}}{\tau_{t}} \frac{\tilde{h}-h_{i} \tilde{Y}_{i}}{c_{p}} \rho \mid \hat{T}\right\rangle=\frac{1}{2} \frac{C_{\phi}}{\tau_{t}}(\tilde{T}-\hat{T}) \rho
$$

if the composition and the specific heat capacity are constant. This corresponds to the IEM model in terms of temperature. The second term under the temperature derivative represents the conditional expectation of heat release. This term describes the effect of chemical reactions on the evolution of $\mathscr{F}_{T}$.

\subsection{Stochastic Representation of MDF}

Due to the high dimensionality of the MDF, numerical solution techniques like finitedifference-schemes can be employed in rare situations only (see e.g. [68]). An efficient way of solving Eq. (6) is to use Lagrangian Monte-Carlo methods as suggested by Pope [81], which require a stochastic representation of the MDF. To this end, the discrete MDF is defined in terms of the Dirac delta-function $\delta$ by

$$
\begin{aligned}
& \mathscr{F}_{N \phi}\left(\hat{h}, \hat{Y}_{1}, \ldots, \hat{Y}_{N_{s}-1}, x_{1}, x_{2}, x_{3} ; t\right)= \\
& \quad \sum_{n=1}^{N_{P}} m_{n} \delta\left(\hat{h}-h^{*(n)}(t)\right) \prod_{i=1}^{N_{s}-1} \delta\left(\hat{Y}_{i}-Y_{i}^{*(n)}(t)\right) \prod_{\alpha=1}^{3} \delta\left(x_{\alpha}-x_{\alpha}^{*(n)}(t)\right)
\end{aligned}
$$

as an ensemble of $N_{P}$ stochastic particles. Every stochastic particle possesses its own individual mass $m_{n}$, position in physical space $x_{\alpha}^{*}$, and its own thermochemical state denoted by its specific enthalpy $h^{*}$ and composition $Y_{i}^{*}$. Each of these particles (which can be thought of to represent samples of the stochastic process, which underlies the MDF) evolves in time according to the following system of stochastic differential equations (SDE)

$$
\begin{aligned}
x_{\alpha}^{*}(t+d t) & =x_{\alpha}^{*}(t)+\left(\tilde{u}_{\alpha}+\frac{1}{\langle\rho\rangle} \frac{\partial}{\partial x_{\alpha}}\left(\langle\rho\rangle D_{T}\right)\right) d t+\sqrt{2 D_{T}} d W_{\alpha}, \\
h^{*}(t+d t) & =h^{*}(t)-\frac{1}{2} \frac{C_{\phi}}{\tau_{t}}\left(h^{*}(t)-\tilde{h}\right) d t, \\
Y_{i}^{*}(t+d t) & =Y_{i}^{*}(t)+\left(\frac{\dot{m}_{i}}{\rho}-\frac{1}{2} \frac{C_{\phi}}{\tau_{t}}\left(Y_{i}^{*}(t)-\tilde{Y}_{i}\right)\right) d t .
\end{aligned}
$$

In these equations, $W_{\alpha}$ denotes a Wiener process, which is the stochastic force. As outlined by Pope [81], the Lagrangian PDF of this system of stochastic particles serves as the transition density of the MDF. In this way stochastic equivalence is established 
between the MDF of the stochastic system and the MDF of the reactive flow, provided that the consistency condition

$$
\left\langle\rho\left(x_{1}, x_{2}, x_{3}, t\right)\right\rangle=\left\langle\sum_{n=1}^{N_{P}} m_{n} \prod_{\alpha=1}^{3} \delta\left(x_{\alpha}-x_{\alpha}^{*(n)}(t)\right)\right\rangle
$$

holds [81]. If the consistency condition is fulfilled, then the expectation of the discrete MDF equals the MDF of the reactive flow [81], i.e.

$$
\mathscr{F}_{\phi}=\left\langle\mathscr{F}_{N \phi}\right\rangle
$$

With the help of the discrete MDF in Eq. (12) Favre averages of thermochemical quantities can be estimated from the properties $\phi_{k}^{*(n)}$ of the stochastic particles as

$$
\tilde{\phi}_{k} \approx \frac{\sum_{n=1}^{N_{p \mathrm{VOL}}} m_{n} \phi_{k}^{*(n)}}{\sum_{n=1}^{N_{p \mathrm{VOL}}} m_{n}}
$$

This estimate is valid for a small, statistically homogeneous volume in physical space which contains $N_{p \mathrm{VOL}}$ stochastic particles [81]. In addition to the Favre averages, the expectations of random variables are required for the comparison to experimental data. If $Q\left(\phi_{k}\right)$ denotes a deterministic function of the random variable $\phi_{k}$, then its expectation can be estimated from the discrete representation of the MDF via

$$
\left\langle Q\left(\phi_{k}\right)\right\rangle \approx\langle\rho\rangle \frac{\sum_{n=1}^{N_{p \mathrm{VOL}}} \frac{m_{n}}{\rho^{*(n)}} Q\left(\phi_{k}^{*(n)}\right)}{\sum_{n=1}^{N_{p \mathrm{VOL}}} m_{n}} .
$$

In this equation, $\rho^{*(n)}$ denotes the density of a stochastic particle, which is computed from its individual thermodynamic state.

\subsection{Solution Algorithm}

In order to solve the system (13)-(15) a hybrid Finite-Volume/Lagrangian Monte-Carlo Method is implemented into the computational-fluid-dynamics (CFD) code THETA of DLR $[62,85]$, which has been already used in earlier PDF simulations of reactive flows $[30,31]$. In this hybrid approach, turbulence time- and length-scales and Favre averaged flow velocities (which are required for the solution of Eqs. (13)-(15)) are provided by the finite-volume solver, where the momentum equations are solved numerically in conjunction with turbulence model equations. The finite volume solver passes its data to the Lagrangian Monte-Carlo method, which returns expectations of density and molecular viscosity to the finite volume solver. Both solvers exchange their data after one iteration until statistical stationarity is reached. In order to pass the data from the finite volume solver to the particles residing in a cell of the finite volume mesh, the "Local Constant Mean Estimate" (LCME) approach [32] is used, which has been employed successfully in earlier work [31]. The system of equations (13)-(15) is integrated in time with the method of fractional steps [81], which is equivalent to a factorization of the MDF transport equation [81]. 


\subsection{Estimation of PDF}

One of the main objectives of the present work is to compare the one-point, onetime marginal PDF of temperature to experimental data (cf. Sec. 4.2). To this end, histograms of temperature are evaluated from the particle data. In order to compute these histograms it is important to consider that according to Eq. (12) the stochastic particles in the Monte-Carlo method represent samples of an MDF, not a PDF. In order do derive the correct evaluation procedure for the particle data, the definition of a histogram is revisited. A histogram $H_{\phi_{i}}\left(\psi_{i}\right)$ of a random variable $\phi_{i}$ is defined as

$$
H_{\phi_{i}}\left(\psi_{i}\right)=\frac{\operatorname{Prob}\left(\psi_{i}+\Delta \psi_{i}>\phi_{i} \geqslant \psi_{i}\right)}{\Delta \psi_{i}},
$$

i.e. as the probability $\operatorname{Prob}$ of a random variable $\phi_{i}$ to lie in the interval $\left[\psi_{i} ; \psi_{i}+\Delta \psi_{i}\right)$, normalized by the width $\Delta \psi_{i}$. As the interval $\Delta \psi_{i}$ approaches zero, the histogram converges towards the marginal PDF of the random variable $\phi_{i}$. The histogram defined above can be expressed in terms of the joint PDF $P_{\phi}(\boldsymbol{\psi})$, and subsequently in terms of the $\operatorname{MDF} \mathscr{F}_{\phi}=\rho P_{\phi}$ as

$$
\begin{aligned}
H_{\phi_{i}}\left(\psi_{i}\right) & =\frac{1}{\Delta \psi_{i}} \int_{\Psi_{1}=-\infty}^{\infty} \ldots \int_{\Psi_{i}=\psi_{i}}^{\psi_{i}+\Delta \psi_{i}} \ldots \int_{\Psi_{N_{\phi}}=-\infty}^{\infty} P_{\phi} d \Psi_{1} \cdots d \Psi_{i} \cdots d \Psi_{N_{\phi}} \\
\Leftrightarrow H_{\phi_{i}}\left(\psi_{i}\right) & =\frac{1}{\Delta \psi_{i}} \int_{\Psi_{1}=-\infty}^{\infty} \ldots \int_{\Psi_{i}=\psi_{i}}^{\psi_{i}+\Delta \psi_{i}} \cdots \int_{\Psi_{N_{\phi}}=-\infty}^{\infty} \frac{\mathscr{F}_{\phi}}{\rho} d \Psi_{1} \cdots d \Psi_{i} \cdots d \Psi_{N_{\phi}} .
\end{aligned}
$$

Entering the definition of the discrete MDF (Eq. (12)) in the above equation yields finally to the following discrete approximation of the histogram

$$
\begin{aligned}
H_{\phi_{i}}\left(\psi_{i}\right) & \approx \frac{1}{\Delta \psi_{i}} \int_{\Psi_{1}=-\infty}^{\infty} \cdots \int_{\Psi_{i}=\psi_{i}}^{\psi_{i}+\Delta \psi_{i}} \cdots \int_{\Psi_{N_{\phi}}=-\infty}^{\infty} \frac{\mathscr{F}_{N \phi}}{\rho} d \Psi_{1} \cdots d \Psi_{i} \cdots d \Psi_{N_{\phi}} \\
\Leftrightarrow H_{\phi_{i}}\left(\psi_{i}\right) & \approx \frac{1}{\Delta \psi_{i}} \sum_{n=1}^{N_{P}} \frac{m_{n}}{\rho^{*(n)}} I_{\left[\psi_{i} ; \psi_{i}+\Delta \psi_{i}\right)}\left(\phi_{i}^{*(n)}\right) \prod_{\alpha=1}^{3} \delta\left(x_{\alpha}-x_{\alpha}^{*(n)}\right) .
\end{aligned}
$$

$\rho^{*(n)}$ denotes the density of a particle as in Eq. (19), and $I_{\left[\psi_{i} ; \psi_{i}+\Delta \psi_{i}\right)}\left(\phi_{i}^{*(n)}\right)$ the indicator function in thermochemical space which is defined as

$$
I_{\left[\psi_{i} ; \psi_{i}+\Delta \psi_{i}\right)}\left(\phi_{i}^{*(n)}\right)= \begin{cases}1, & \text { if } \phi_{i}^{*(n)} \in\left[\psi_{i} ; \psi_{i}+\Delta \psi_{i}\right) \\ 0, & \text { otherwise }\end{cases}
$$

This indicator function is an artifact of the sifting property of the Dirac-function and means essentially that only particles are considered in the histogram, whose composition lies in the range of the histogram-bin. In order to obtain a workable expression for the histogram, the last expression is averaged over a small, statistically homogeneous 
volume $\Delta V=\Delta x_{1} \times \Delta x_{2} \times \Delta x_{3}$ in physical space [81] which yields finally to

$$
\begin{aligned}
& H_{\phi_{i}}\left(\psi_{i}\right) \approx \\
& \frac{1}{\Delta \psi_{i} \Delta V} \sum_{n=1}^{N_{P}} \frac{m_{n}}{\rho^{*(n)}} I_{\left[\psi_{i} ; \psi_{i}+\Delta \psi_{i}\right)}\left(\phi_{i}^{*(n)}\right) \prod_{\alpha=1}^{3} I\left[x_{\alpha} ; x_{\alpha}+\Delta x_{\alpha}\right)\left(x_{\alpha}^{*(n)}\right) .
\end{aligned}
$$

Here $I_{\left[x_{\alpha} ; x_{\alpha}+\Delta x_{\alpha}\right)}\left(x_{\alpha}^{*(n)}\right)$ denotes the indicator function in physical space, which is given by

$$
I_{\left[x_{\alpha} ; x_{\alpha}+\Delta x_{\alpha}\right)}\left(x_{\alpha}^{*(n)}\right)=\left\{\begin{array}{ll}
1, & \text { if } x_{\alpha}^{*(n)} \in\left[x_{\alpha} ; x_{\alpha}+\Delta x_{\alpha}\right) \\
0, & \text { otherwise }
\end{array} .\right.
$$

Equation (20) is the correct approach for computing the histogram from the particle representation of the MDF. It differs from the usual definition of a histogram due to the additional factor $m_{n} / \rho^{*(n)}$, which may be interpreted as an individual volume occupied by a particle. Finally, it is still necessary to determine $\Delta V$ for the evaluation of the histogram. An elegant approach is to use the identity

$$
\Delta V=\int_{\Psi_{1}=-\infty}^{\infty} \cdots \int_{\Psi_{N_{\phi}}=-\infty}^{\infty} \int_{\hat{x}_{1}=x_{1}}^{x_{1}+\Delta x_{1}} \int_{\hat{x}_{2}=x_{2}}^{x_{2}+\Delta x_{2}} \int_{\hat{x}_{3}=x_{3}}^{x_{3}+\Delta x_{3}} \frac{\mathscr{F}_{\phi}}{\rho} d \hat{x}_{1} d \hat{x}_{2} d \hat{x}_{3} d \Psi_{1} \cdots d \Psi_{N_{\phi}}
$$

and to apply this equation to the discrete MDF. This yields finally to the simple expression

$$
\Delta V \approx \sum_{n=1}^{N_{P}} \frac{m_{n}}{\rho^{*(n)}} \prod_{\alpha=1}^{3} I\left[x_{\alpha} ; x_{\alpha}+\Delta x_{\alpha}\right)\left(x_{\alpha}^{*(n)}\right) .
$$

This is a useful definition of the volume since it ensures that the integral of the histogram given by Eq. (20) over the variable $\psi_{i}$ is equal to one. Thus, equations (20) and (21) jointly provide a method for computing the histogram from particle data.

One important detail which has not been mentioned so far is the exact definition of the random vector $\phi_{i}$. If we are interested in the histogram of temperature, the above derivation implies that we need the joint MDF of temperature and composition, i.e. $\mathscr{F}_{T, Y_{i}}\left(\hat{T}, \hat{Y}_{i}\right)$, but we are modeling a joint MDF of specific enthalpy and composition $\mathscr{F}_{h, Y_{i}}\left(\hat{h}, \hat{Y}_{i}\right)$. This is, however, not a problem, since both MDFs are related to each other via Eq. (7). The above integration over temperature transforms into an integration over enthalpy, and the MDF $\mathscr{F}_{T, Y_{i}}\left(\hat{T}, \hat{Y}_{i}\right)$ can be substituted by $\mathscr{F}_{h, Y_{i}}\left(\hat{h}, \hat{Y}_{i}\right)$. Hence, in order compute the histogram of temperature from the particle data according to Eq. (20), the temperature of each particle must be calculated from its individual enthalpy and composition. 


\subsection{Computational Model}

Since we are interested in the simulation of a steady lifted flame, steady state simulations are performed here. In order to model turbulence, the two equation standard $k-\varepsilon$ model of Jones and Launder [48] is used. As in previous work [31], the $C_{\varepsilon 1}$ model constant is increased to a value of $C_{\varepsilon 1}=1.53$ in order to correct the round jet anomaly of this model. The remaining constants are kept at the values given by Launder and Sharma [57]. Figure 2 shows the computational domain. Due to the rotational symmetry of the jet, only a $10^{\circ}$ segment of the jet is considered. To model the coflow, a constant stream of burnt gases is prescribed at the coflow boundary shown in Fig. 2. The flow direction in Fig. 2 is parallel to the x-axis. The thermochemical state, the flow velocity, and the turbulence time and length scale are taken to be uniform at the coflow boundary. A turbulence intensity of $5 \%$ is assumed and the turbulence length scale is taken to be $7 \%$ of the hydraulic diameter of the chamber [99]. All stochastic particles entering the domain through the coflow boundary have the same temperature and composition. The coflow temperature is measured $40 \mathrm{~mm}$ above the nozzle exit plane at a radial distance of $15 \mathrm{~mm}$ from the jet axis. Its composition is taken to be at chemical equilibrium and is computed as outlined in $[7,75]$. In order to obtain a fully developed pipe flow at the jet exit plane, a section of the pipe with 50 diameters length is included in the computational domain. At the inflow of the pipe, the average velocity is prescribed. As stated before, the length scale of turbulence is assumed to be $7 \%$ of the hydraulic diameter and the turbulence intensity is taken to be $10 \%$ [99]. The stochastic particles entering the domain at the pipe inflow have the same thermochemical state. As indicated in Fig. 2, the finite width of the fuel pipe is included in the computational domain as well as the distance between the nozzle exit plane and the plate of the matrix burner. The computational mesh consists of 50,289 cells and 101,693 grid points. To resolve the jet 19 cells are distributed across the radius (i.e. 38 cells for the whole diameter). For the modeling of the MDF 128 particles per cell are employed in the present simulation. Based on earlier work with the same method $[30,31]$, where the effect of particle number is studied, this number of particles is sufficient for this kind of simulation. To incorporate the effect of chemical kinetics GRI 3.0 reaction mechanism [87] is used in this work with 52 species and 323 reactions.

In order to check the validity of the reaction mechanism under the present conditions, calculated ignition delay times are compared to measurements of Petersen et al. [79].

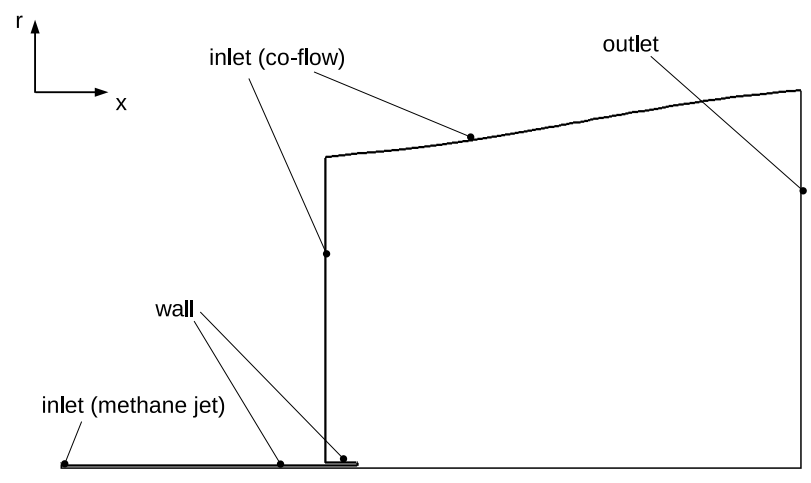

Figure 2. Computational domain. 


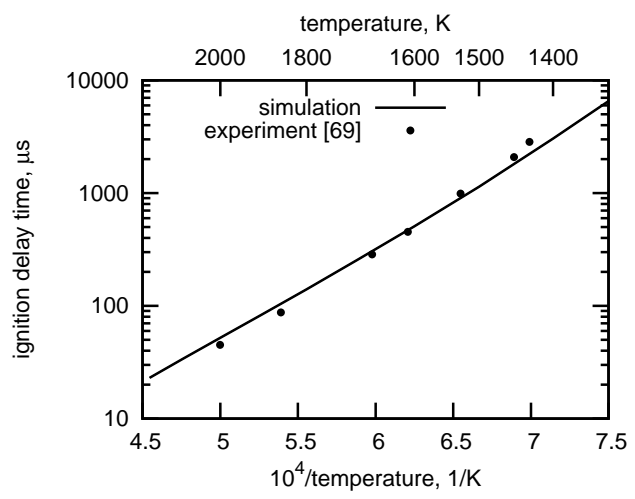

Figure 3. Ignition delay times of GRI 3.0 reaction mechanism (experimental data from [79]).

In [79], shock-tube data are given for the ignition delay time of a lean methane-air mixture (equivalence ratio of 0.5 ) for different temperatures at a pressure of $0.72 \mathrm{~atm}$. To compute the ignition delay time, adiabatic constant volume reactors are simulated using Cantera [37]. The results are summarized in Fig. 3, where in general a good agreement to the measurements of Petersen et al. [79] is found over the whole temperature range. However, in the relevant region between $1400 \mathrm{~K}$ and $1500 \mathrm{~K}$, where the auto-ignition of the methane jet is expected to occur in the DLR-JHC burner, some deviations are observed. Here, the computation with the GRI 3.0 mechanism results in a shorter ignition delay time compared to the experiment. Thus, a slightly shorter lift-off height is to be expected in the TPDF calculation due to the reaction mechanism.

\section{Results and Discussion}

\subsection{First and Second Moments}

A first comparison between computational and experimental data is given in this section in terms of first and second moments. Averages and variances are computed from the particle data by the use of Eq. (19). Time averaging is used to reduce the stochastic error (the estimates for averages and variances are uniformly averaged over the last 100 iterations in the statistically stationary state).

\subsubsection{Mixture Fraction}

The capability of the model to capture the mixing of the reactants is investigated first. To this end, results for average mixture fraction and mixture fraction variance are presented. As outlined in Sec. 2.2, planar Rayleigh scattering is used to determine mixture fraction which is only possible in regions where no chemical reactions occur. Hence, only data "below" the lifted flame, i.e. at $X=10 \mathrm{~mm}, \mathrm{X}=20 \mathrm{~mm}, \mathrm{X}=30 \mathrm{~mm}$, and $\mathrm{X}=40 \mathrm{~mm}$ are shown in Figs. 4 and 6 . If we compare first the measured and computed averages of mixture fraction in Fig. 4 we find good agreement at $\mathrm{X}=40 \mathrm{~mm}$. As we move upstream, however, the agreement becomes poorer at a radius of $0 \mathrm{~mm}$. At $10 \mathrm{~mm}$ finally the value of the simulation is $37 \%$ higher than the experimental value. The radial width of the profiles, however, is captured surprisingly well in the simulation. In order to highlight the deviations, a profile of average mixture fraction 


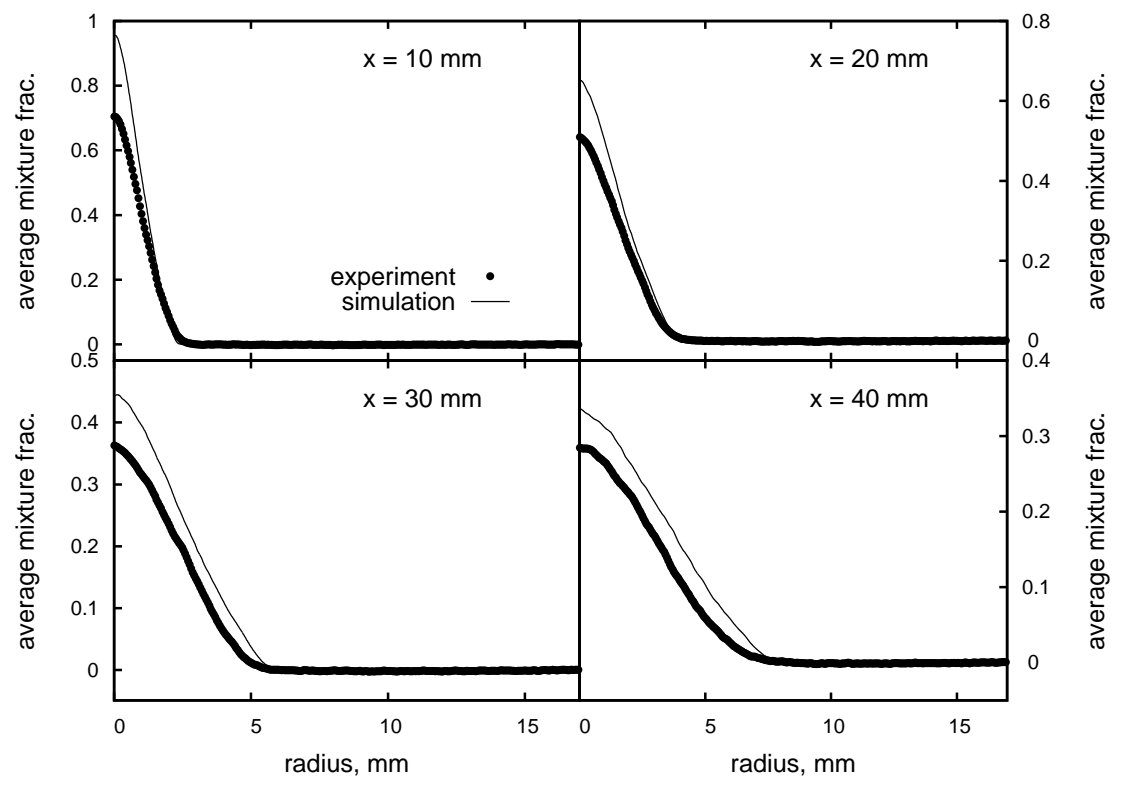

Figure 4. Average mixture fraction.

is shown along the center line (i.e. at a radius of $0 \mathrm{~mm}$ ) in Fig. 5. This center line profile shows that the discrepancy between simulation and experiment increases as one moves upstream towards the nozzle exit plane. In order to shed light on this problem, the analytic correlation

$$
Z=5.3 Z_{0} \sqrt{\frac{\rho_{j}}{\rho_{c}}} \frac{1}{\frac{X}{d}+0.85}
$$

provided by Talbot et al. [93] is used in this work to provide an additional reference. In this equation $Z$, denotes the mixture fraction, $Z_{0}$ the mixture fraction at the nozzle exit (which is one), $\rho_{j}$ the density of the jet, $\rho_{c}$ the density of the coflow, and $d$ the nozzle diameter. Equation (22) is derived from experimental data and describes the evolution of a passive scalar (in the present case, mixture fraction) along the center line of a turbulent, round, non-reacting jet. The curve "literature" in Fig. 5 represents the mixture fraction profile for the present test case. These data from literature are in excellent agreement with the results of the present simulation whereas the experimental data clearly deviate from this analytic expression. It should be noted, however, that

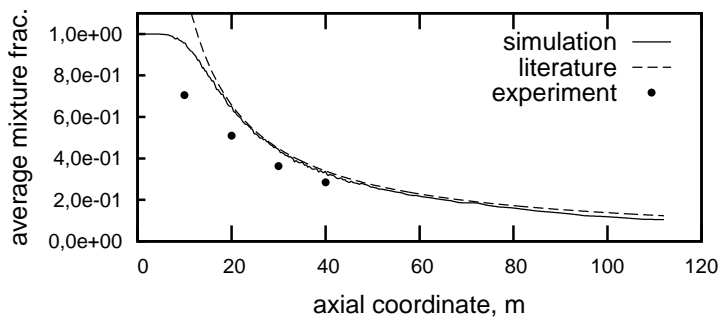

Figure 5. Average mixture fraction along center line. 


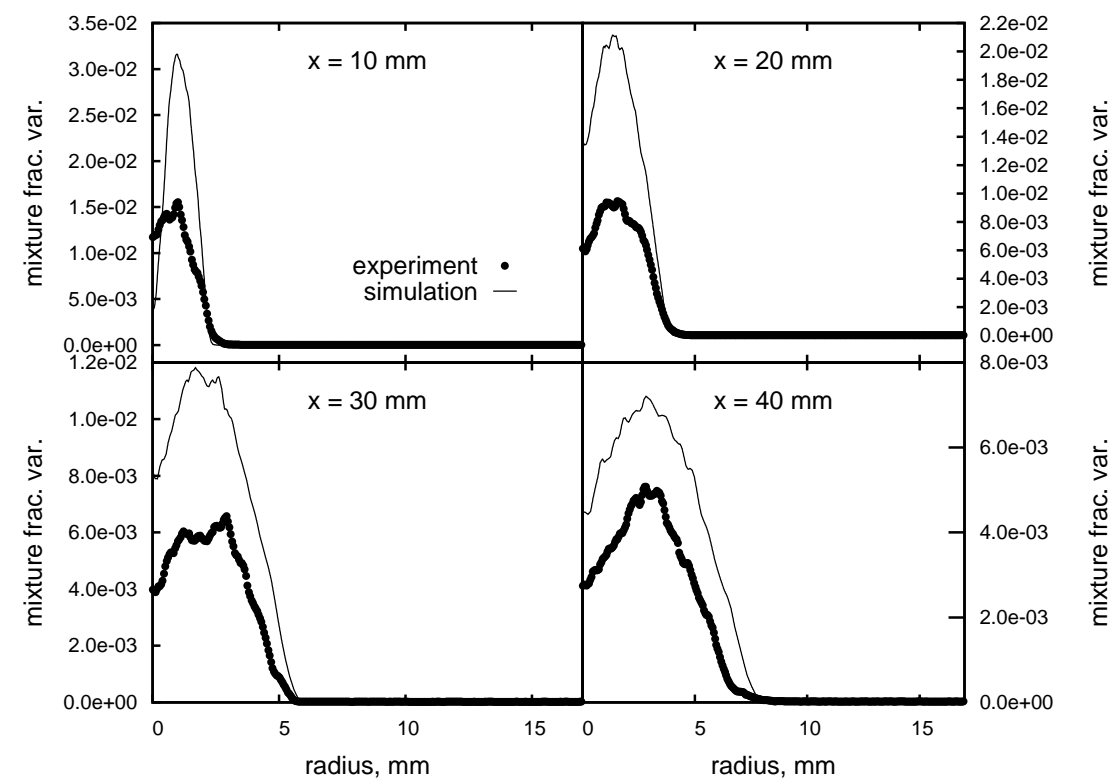

Figure 6. Mixture fraction variance.

Talbot et al. [93] use a significantly larger nozzle in their experiments with an inner diameter of $5 \mathrm{~mm}$. The nozzle in the present experiment has a diameter of $1.5 \mathrm{~mm}$. It is unclear if the empirical correlation given by Talbot et al. [93] can be extrapolated to very small nozzles. To our knowledge, no other experiments at such small nozzle diameters are available for reference. A possible explanation for the deviations in the present experiment is the finite width of the nozzle walls, which is large compared to the diameter. The outer diameter of the nozzle amounts to $3 \mathrm{~mm}$ [9], i.e. the nozzle has a wall thickness of $0.75 \mathrm{~mm}$. Additionally the end of the nozzle is flat. Hence, there is a possibility that a vortex is formed on the rim, which may increase the initial spreading rate as outlined by Kollmann and Janicka [55]. The present $k-\varepsilon$ turbulence model cannot predict such an effect [55].

Lastly, the variance of mixture fraction is given in Fig. 6. Due to the overestimation of the mean value at $\mathrm{X}=10 \mathrm{~mm}$ and $\mathrm{X}=20 \mathrm{~mm}$, the variances of mixture fraction are over predicted by the simulation too at these positions. However, the qualitative shape of the profiles is captured very well. In particular, the positions of the maxima in mixture fraction variance are accurately reproduced. These maxima are found in regions where the largest gradients of average mixture fraction exist (i.e. where the largest degree of unmixedness is found). At $\mathrm{X}=30 \mathrm{~mm}$ and $\mathrm{X}=40 \mathrm{~mm}$, the absolute values of computed and measured mixture fraction variances agree slightly better. As in the upstream positions, the qualitative shape is computed accurately.

\subsubsection{Lift-off Height}

Next, the lift-off height of the flame is analyzed. In contrast to previous studies [9], the lift-off height here is not defined as the average position of the lower flame edge; instead it is defined in terms of $\mathrm{OH}$ concentration, which is a more convenient definition for a comparison to RANS (Reynolds Averaged Navier-Stokes) simulations. The approach is illustrated in Fig. 7, where the lift-off height $(\mathrm{LOH})$ is defined as the distance between the nozzle exit plane and the earliest point in the flame where twice the coflow $\mathrm{OH}$ 

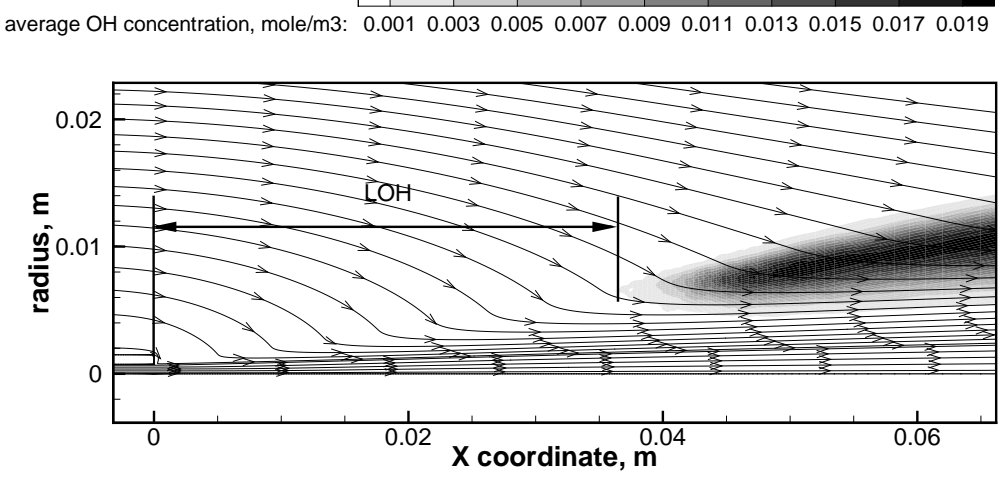

Figure 7. Computed lift-off height. Color coded is the $\mathrm{OH}$ concentration (in $\mathrm{mole} / \mathrm{m}^{3}$ ) and overlaid are streamlines of velocity.

concentration is reached first. The average $\mathrm{OH}$ concentration is computed via Eq. (19) from the data of the stochastic particles. In order to reduce the stochastic error, particle averages are estimated for the last 100 iterations in the statistically stationary state of the simulation. An average over these 100 individual fields is then computed, which reduces the stochastic noise. If we use the aforementioned definition of lift-off height, a lift-off height of $36 \mathrm{~mm}$ is found in the simulation. Due to a slight asymmetry in the experiment, the lift-off height varies between 42 and $45 \mathrm{~mm}$. This corresponds to a deviation between simulation and experiment from $14 \%$ to $22 \%$. The shorter lift-off height in the simulation is probably caused by the reaction mechanism, which tends to underestimate the ignition delay time for these conditions (cf. Sec. 3.5).

\subsubsection{Hydroxyl}

After the previous qualitative comparison, the $\mathrm{OH}$ concentration profiles are investigated quantitatively next. Profiles of the mean and variance of $\mathrm{OH}$ concentration are shown in Fig. 8 and Fig. 9 respectively. These profiles are extracted at four different distances away from the nozzle exit plane, i.e. at $\mathrm{X}=30 \mathrm{~mm}, \mathrm{X}=40 \mathrm{~mm}, \mathrm{X}=50 \mathrm{~mm}$, and $\mathrm{X}=60 \mathrm{~mm}$. The measurement uncertainties reported in Sec. 2.2 are shown in terms of error bars. The best agreement between measured and computed average value of $\mathrm{OH}$ concentration is found at a distance of $\mathrm{X}=60 \mathrm{~mm}$. Here, the shape and the maximum values of the computed and measured profiles match each other very well in particular if the experimental uncertainties are taken into account. This good agreement is not surprising since the flame reaches its fully burning state at this location, as seen in the OH-contour plot in Fig. 7. Thus, slight differences between computed and measured lift-off height do not result in major discrepancies between experiment and simulation at this location. The profiles of computed and measured variance shown in Fig. 9 also agree well in shape at $\mathrm{X}=60 \mathrm{~mm}$, although the simulation overestimates the variance observed in the experiment. Further upstream, however, at $\mathrm{X}=40 \mathrm{~mm}$ and $\mathrm{X}=50 \mathrm{~mm}$ the agreement between experiment and simulation deteriorates. This is due to the difference between computed and measured lift-off height of the flame. Although this difference appears to be small, it still causes major deviations in average $\mathrm{OH}$-concentration at $\mathrm{X}=40 \mathrm{~mm}$ and $\mathrm{X}=50 \mathrm{~mm}$. Due to ignition a large streamwise 


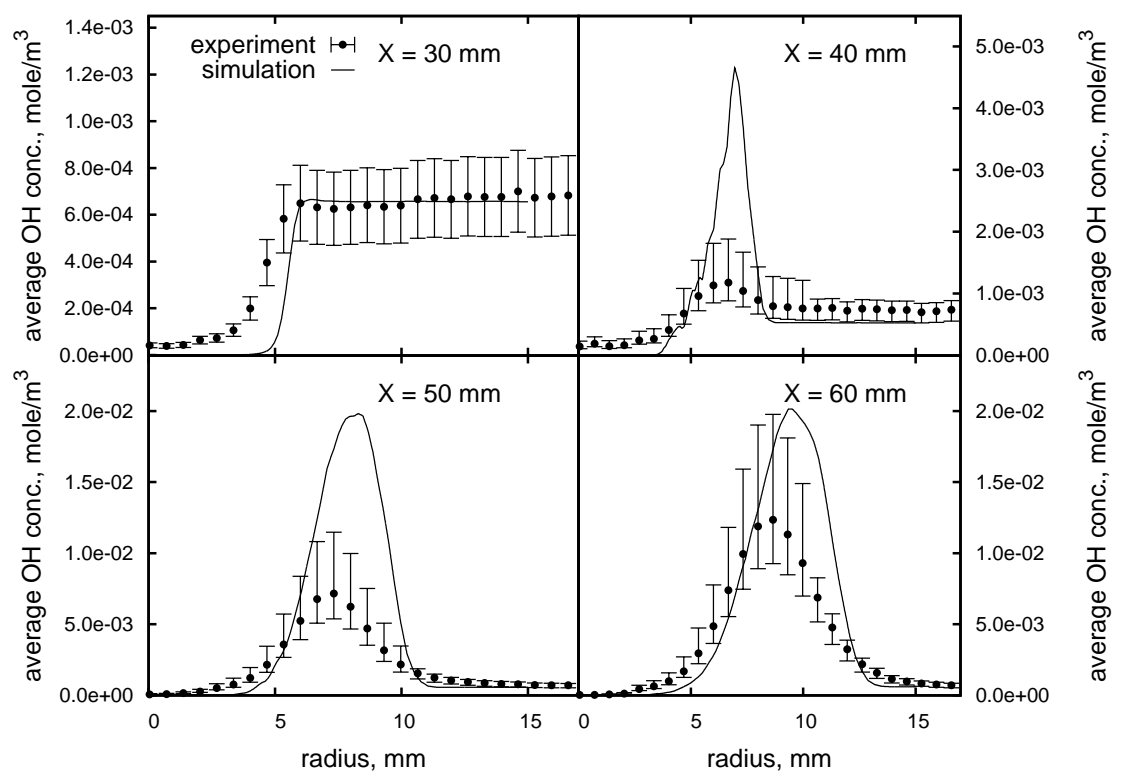

Figure 8. Average hydroxyl concentration.

gradient of average $\mathrm{OH}$ concentration exists between $\mathrm{X}=40 \mathrm{~mm}$ and $\mathrm{X}=50 \mathrm{~mm}$ as can be ascertained from Fig. 7 . Since the simulation predicts a slightly smaller lift-off height compared to the experiment, the rise in $\mathrm{OH}$-concentrations commences further upstream, and hence leads to an overestimation of the average $\mathrm{OH}$ concentration in this area (cf. Fig. 8). Despite the differences in magnitude of average $\mathrm{OH}$ concentration, the qualitative shape of the measured $\mathrm{OH}$ profiles is reproduced well by the simulation. Moreover, the position at which the maxima of average $\mathrm{OH}$ concentration

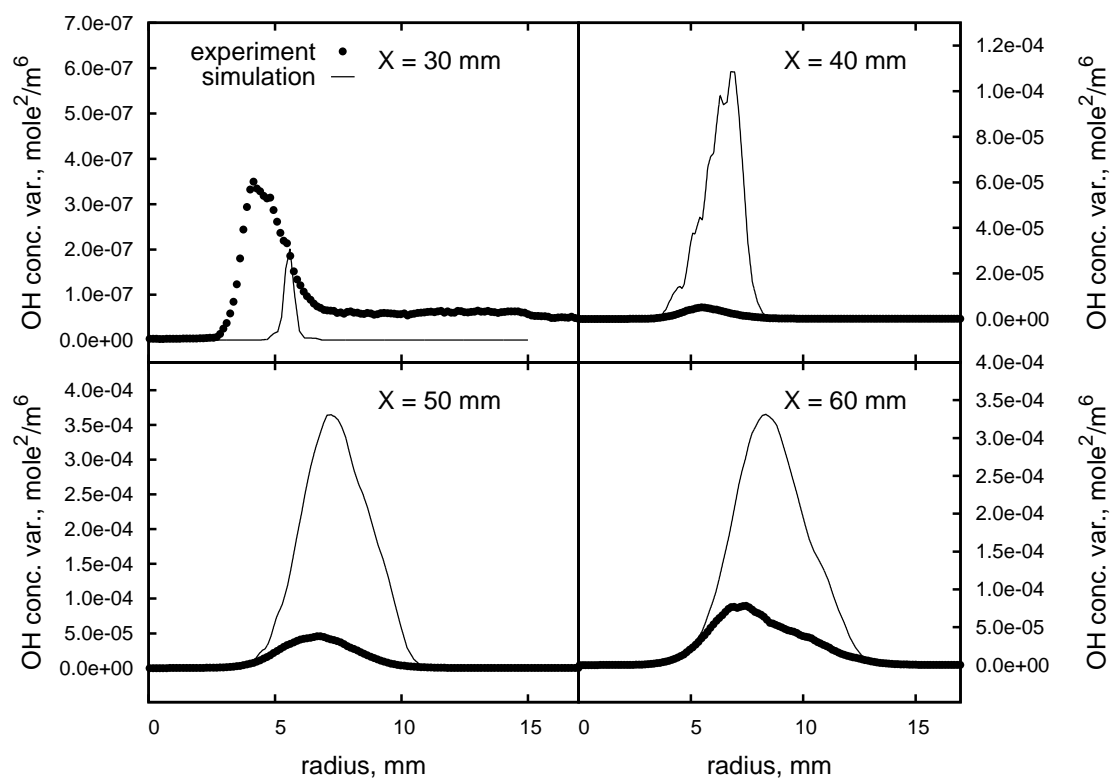

Figure 9. Hydroxyl concentration variance. 
occur is also captured very well. At $\mathrm{X}=30 \mathrm{~mm}$, no stable flame is observed. Computed and measured averages agree very well here.

Due to the differences in lift-off height the computed variances at $\mathrm{X}=40 \mathrm{~mm}$ and $\mathrm{X}=50 \mathrm{~mm}$ shown in Fig. 9 do not match the experimental data in magnitude. The radial position and the qualitative form of the $\mathrm{OH}$ variance profiles are, however, reproduced very well in the simulation. At $\mathrm{X}=30 \mathrm{~mm}$ the agreement between computed and measured variance is poor. This deviation could be caused by experimental uncertainties in the estimation of the variance: the variance in the coflow must be zero, which (at this location) is not the case in the experiment.

\subsubsection{Temperature}

In Figs. 10 and 11 a comparison is given between measured and calculated mean and variance of temperature. The profiles shown in these figures are extracted at $X=20 \mathrm{~mm}, X=30 \mathrm{~mm}, X=40 \mathrm{~mm}$, and $X=50 \mathrm{~mm}$. In Fig. 10, the ignition of the fuel is clearly visible at $\mathrm{X}=50 \mathrm{~mm}$ due to the temperature rise above the coflow value of $1490 \mathrm{~K}$. Despite the fact that the maximum temperature at $\mathrm{X}=50 \mathrm{~mm}$ is slightly over predicted in the simulation, the overall agreement between simulation and experiment is good. The position of the temperature peaks and the shape is reproduced accurately at $\mathrm{X}=50 \mathrm{~mm}$. At the further upstream position in Fig. 10, no temperature rise due to ignition is visible in the averages. In this area the agreement between simulation and experiment is excellent. A good agreement between simulation and experiment is also found in temperature variance (cf. Fig. 11). The temperature variance reaches its maximum value in the shear layer where the largest gradients of mean temperature exist. Only at $\mathrm{X}=50 \mathrm{~mm}$ the simulation is not capable to reproduce the absolute magnitude of temperature variance.

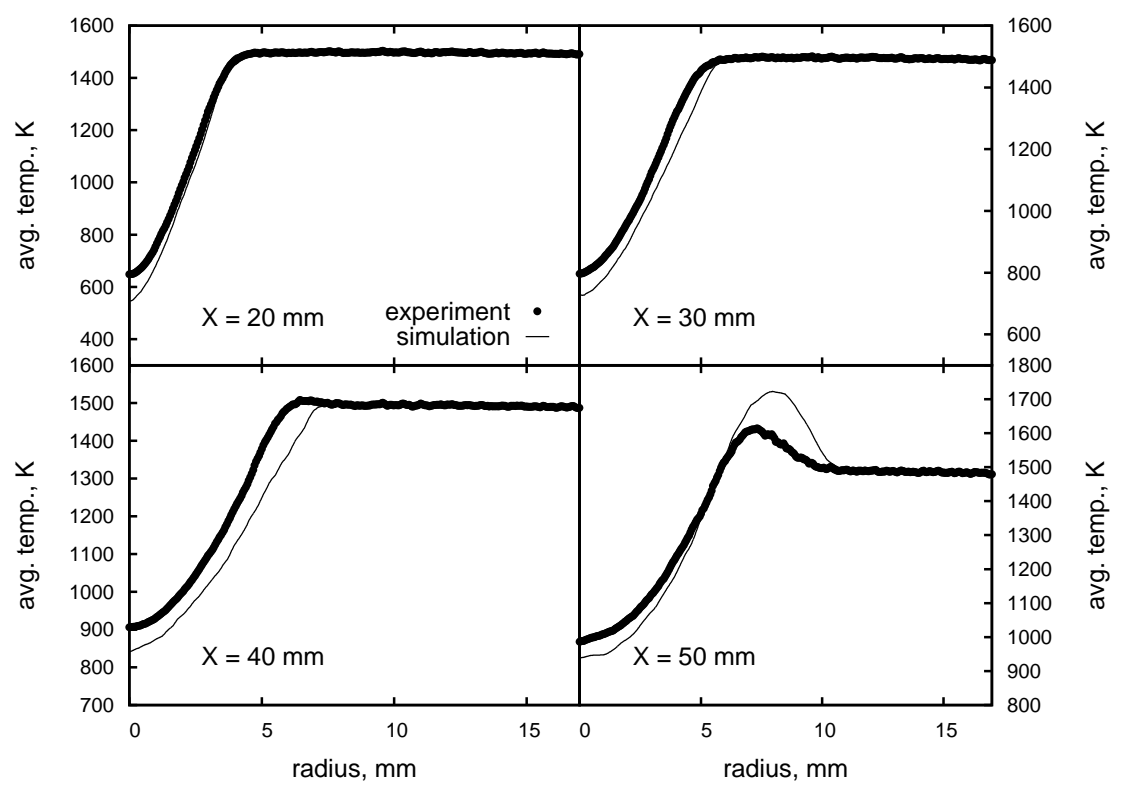

Figure 10. Average temperature. 


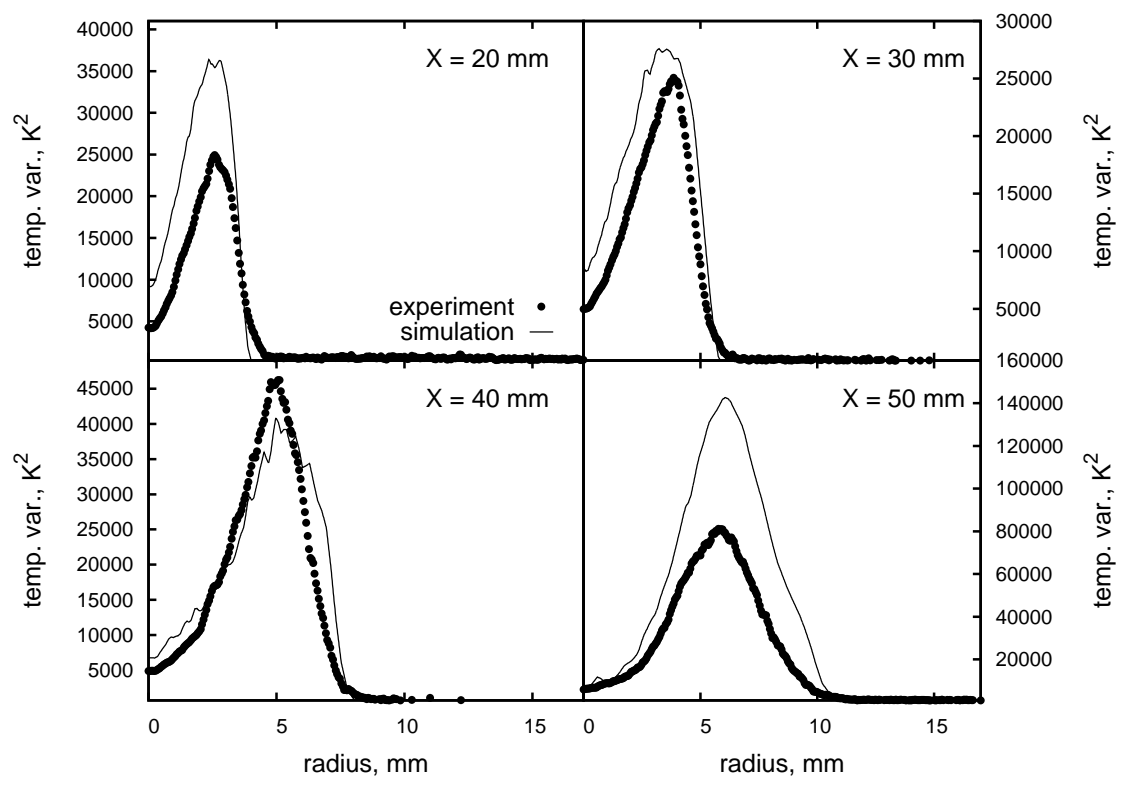

Figure 11. Temperature variance.

\subsection{Marginal PDF of Temperature}

In the following section the evolution of the one-point, one-time marginal PDF of temperature is investigated. The PDF of temperature is important because the occurrence of high temperatures (in conjunction with sufficiently low scalar dissipation rate) can trigger auto-ignition even if these events are statistically rare. Hence, the ability of the TPDF method to actually capture the PDF of temperature is of great importance and is investigated here. Histograms of temperature are computed from particle data as outlined in Sec. 3.4. In order to reduce statistical noise in the histograms, time averaging is applied. To this end, the solver is run for 100 more iterations in the statistically stationary state of the solution. Particle data are written for each iteration and for each of these data sets an individual histogram is computed. These individual histograms are then used to compute an average histogram. The result of this procedure is summarized in Figs. 12, 13, 14, and 15. In addition to the simulation data, experimental data (dashed lines) are included in these Figures. In contrast to other works $[55,82]$, where the computed PDFs are extracted at locations where the experimental and computed mean-values coincide (which is not necessarily at the same point in space), the computed and measured PDFs are evaluated here at the same location in physical space. These one point statistics are extracted at the same positions as the average and variance of temperature shown in Figs. 10 and 11 (the PDFs for $\mathrm{X}=20 \mathrm{~mm}$ are given in Fig. 12, for $\mathrm{X}=30 \mathrm{~mm}$ in Fig. 13, for $\mathrm{X}=40 \mathrm{~mm}$ in Fig. 14, and for $\mathrm{X}=50 \mathrm{~mm}$ in Fig. 15). For each of these downstream positions, four radial locations are chosen in the experiment in order to extract temperature PDFs. The first location is always chosen to lie on the center line at a radius $r$ of $r=0 \mathrm{~mm}$. The remaining three positions are distributed in the turbulent shear layer between the jet and coflow. The last of these positions is always closest to the coflow. Since the jet is spreading further as one moves downstream, different radial locations are chosen for each axial position. 


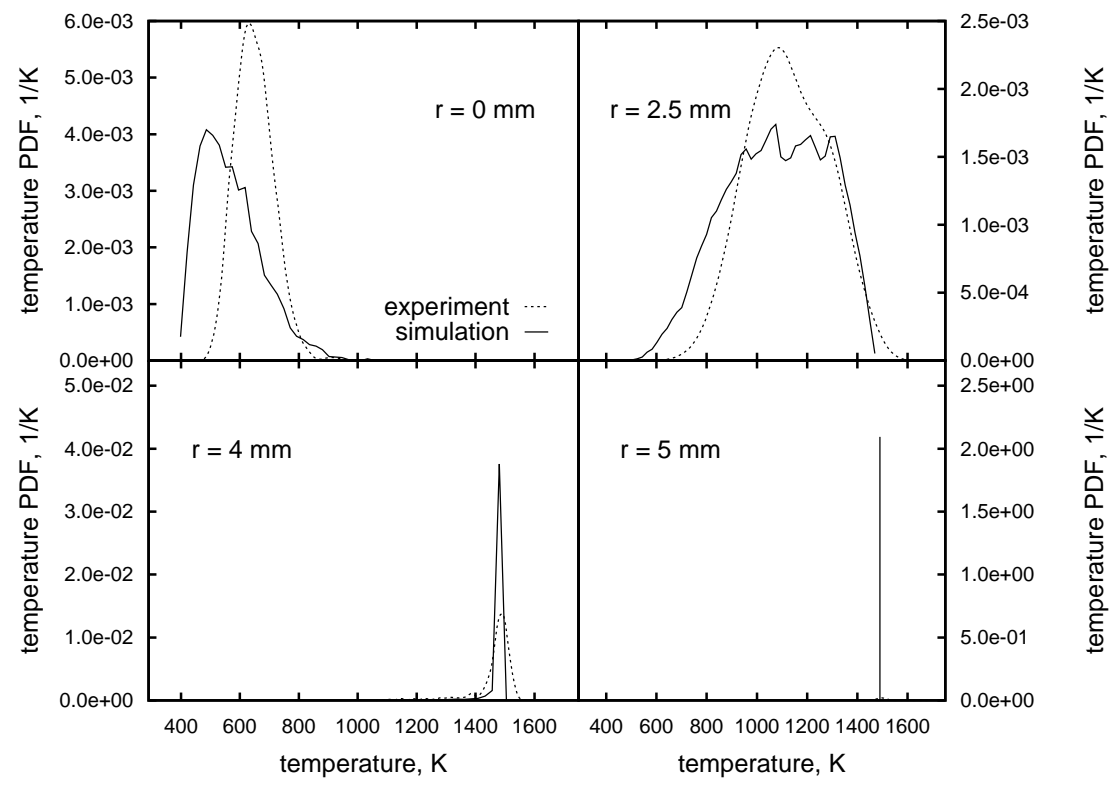

Figure 12. $\mathrm{PDF}$ of temperature at $\mathrm{X}=20 \mathrm{~mm}$.

At an axial distance of $\mathrm{X}=20 \mathrm{~mm}$ no stable flame is observed in the experiment. Hence, temperature may be viewed as a passive scalar in this area. The development of the temperature PDF (cf. Fig 12) resembles the observations of Batt [11] in a planar non-reacting shear layer (which are confirmed by simulations in [55, 82], as outlined by Mungal and Dimotakis [69]). Starting from a free-stream Dirac function at a radius of $5 \mathrm{~mm}$ (i.e. in the hot coflow), the PDF becomes broader and its peak "moves" towards lower temperatures. At $r=4 \mathrm{~mm}$, the PDF is asymmetric with a long tail towards low temperatures and a maximum close to the coflow temperature, which indicates that low temperature fluid of the central jet begins to mix with the hot coflow gases. The PDF of simulation and experiment agree very well at this location. If we move further towards the jet center, the peak of the PDF is shifted towards low temperatures and the PDF becomes broader and symmetric. In the simulation, the PDF appears to be flatter than in the experiment and stretches further towards low temperatures. The qualitative shape, however, is well reproduced. On the axis finally, the PDF appears to be "flipped". The maximum of the PDF is now at low temperatures with a long tail towards high temperatures. The maximum value of the PDF in the simulation is located at a temperature lower than in the experiment, which is consistent with the observation that the mean temperature is slightly underestimated in the simulation (cf. Fig. 10). The shapes of simulated and measured PDF agree, however, very well. A further noteworthy observation is that the PDF at this axial distance are uni-modal. Intermittency as in the experiments of Venkataramani et al. [98] is not observed here.

Further downstream, at $\mathrm{X}=30 \mathrm{~mm}$ a stable flame is still not established. The development of the PDF across the shear layer, which is shown in Fig. 13, is similar to the PDF behavior at $\mathrm{X}=20 \mathrm{~mm}$. Starting from a free-stream Dirac function at $r=6 \mathrm{~mm}$, the PDF develops a long tail towards low temperatures at $r=5 \mathrm{~mm}$. An interesting observation at $r=5 \mathrm{~mm}$ is the presence of temperatures above $1800 \mathrm{~K}$ in the experiment. This could indicate that ignition kernels are present in the 


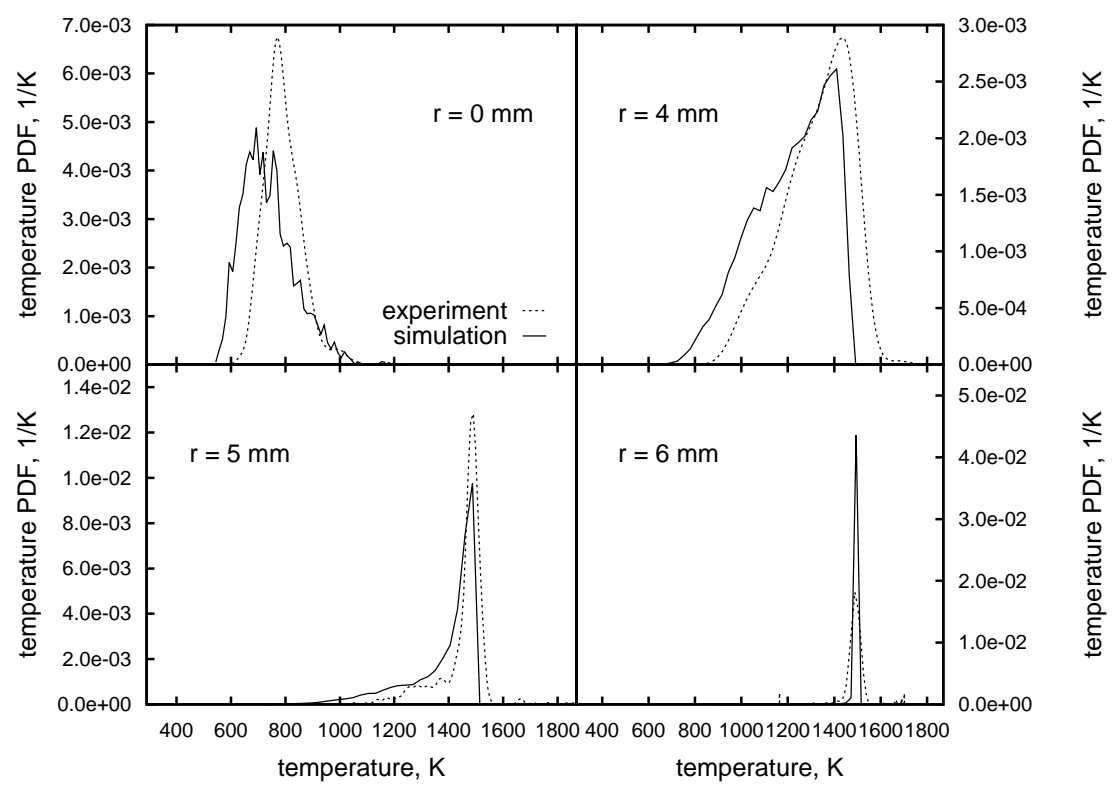

Figure 13. $\mathrm{PDF}$ of temperature at $\mathrm{X}=30 \mathrm{~mm}$.

experiment, although with a very low probability. The same is observed at $r=4 \mathrm{~mm}$. The maximum of the PDF is still close to the coflow temperature. Low temperatures, however, occur now with a higher probability, which indicates a stronger mixing between cold and hot gases and this is captured accurately by the simulation. On the axis at $r=0 \mathrm{~mm}$, the PDF is again asymmetric with its maximum located at cold temperatures. Due to the lower mean value in the simulation, the maximum of the computed PDF is at lower temperatures compared to the experiment. The qualitative shape is, however, well reproduced.

Changes in the development of the PDF across the shear layer start to occur at $\mathrm{X}=40 \mathrm{~mm}$, where chemical reactions start to affect the development of the PDF. Starting again close to the outer edge of the shear layer between jet and coflow (i.e. at $r=8 \mathrm{~mm}$ ), we observe (in addition to the expected free-stream Dirac function) for the first time a tail in the high temperature region. This tail stretches beyond $2000 \mathrm{~K}$. These "high temperature" events are also observed at $r=6.5 \mathrm{~mm}$, where the PDF exhibits still a dominant free-stream Dirac function. At $r=5 \mathrm{~mm}$ we observe a bi-modal form of the PDF in the experiment. A second distinctive maximum is formed due to the ignition at a temperature of $1784 \mathrm{~K}$. Also, in the simulation a bi-model form is observed. It appears, however, that the burnt state is reached faster in the simulation compared to the experiment. Instead of forming a maximum at $1784 \mathrm{~K}$, a peak value is found at the end of the temperature interval at $2092 \mathrm{~K}$, i.e. at the fully burnt state. The shape of the PDF between $800 \mathrm{~K}$ and $1600 \mathrm{~K}$ is, however, reproduced very well in the simulation. On the center-line at $r=0 \mathrm{~mm}$, no reacting states are observed and the PDF is bell shaped in both the simulation and the experiment (where the peak value of the computed PDF being shifted slightly towards lower temperatures).

At the most downstream location of $\mathrm{X}=50 \mathrm{~mm}$ (cf. Fig. 15), the effect of chemical reactions on the PDF is most noticeable. Four maxima in temperature are observed in the experiment at $r=6 \mathrm{~mm}$. Two Dirac functions are present at the end of the 


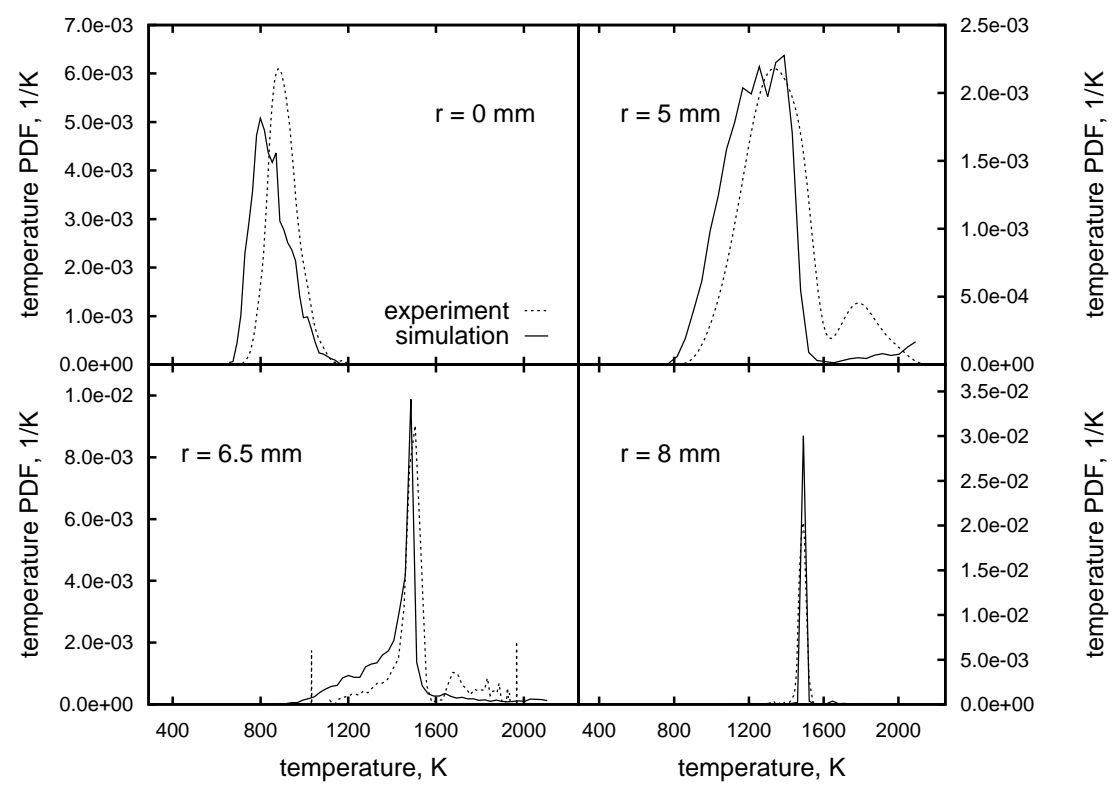

Figure 14. $\mathrm{PDF}$ of temperature at $\mathrm{X}=40 \mathrm{~mm}$.

temperature interval, whereas two maxima are found in the inner of the temperature interval. The Dirac pulses located at the lower and upper ends of the temperature interval are artifacts of data processing in the experiment and should be discarded. Comparing the PDF of the experiment to the simulation, high temperatures seem to occur with a lower probability in the experiment. The PDF in the simulation increases monotonically, whereas an extremum is found in the experiment at $1852 \mathrm{~K}$. The main reason for this deviation is most probably the IEM mixing model. Earlier TPDF

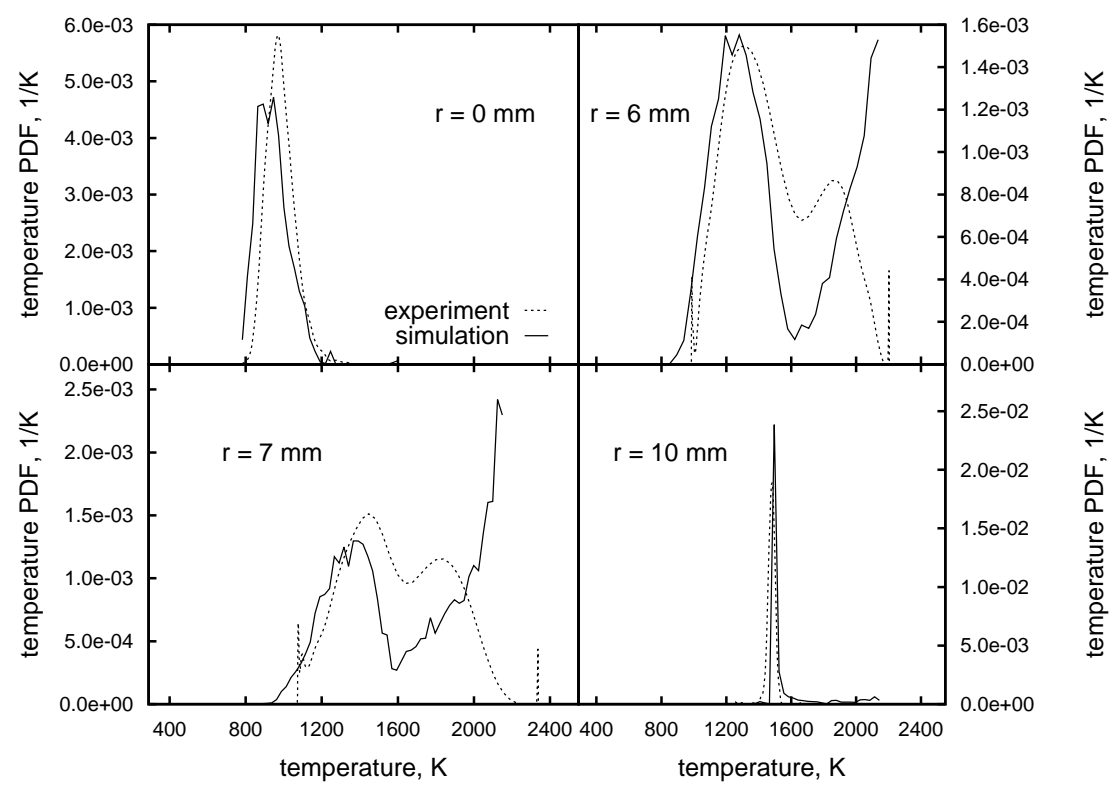

Figure 15. $\mathrm{PDF}$ of temperature at $\mathrm{X}=50 \mathrm{~mm}$. 
computations of lifted flames (see e.g. [19]) already demonstrate that the IEM model tends to lead to bi-model distributions in temperature (burnt and unburnt states). It is shown in [19] that the use of other micro mixing models like the EuclideanMinimum-Spanning-Trees (EMST) model [92] or the modified Curl model [47] leads to improvements in the computation of the temperature distribution in auto-ignition stabilized flames. Moreover, the approach used to model turbulence may also play a significant role, because the turbulence time scale enters directly into the micro mixing model. It is therefore expected that the use of a joint velocity-composition PDF will affect the prediction of the marginal PDF of temperature.

At $r=7 \mathrm{~mm}$, similar behavior is found. Auto-ignition results in a bi-modal PDF in the experiment (the Dirac functions at the end of the temperature range (at $1077 \mathrm{~K}$ and $2336 \mathrm{~K}$ ) are as before artifacts of the data processing procedure in the experiment). The first maximum between $1000 \mathrm{~K}$ and $1600 \mathrm{~K}$ is well reproduced by the simulation. The second maximum in the experiment above $1600 \mathrm{~K}$, however, is not resolved. In the simulation, the PDF reaches its maximum at the end of the temperature interval. The strong increase in the PDF indicates that these burnt states occur with very high probability in the simulation. This also explains the higher mean temperature which is found in Fig. 10 at $\mathrm{X}=50 \mathrm{~mm}$. At the edge of the shear layer $(r=10 \mathrm{~mm})$, the usual free stream Dirac function is found again as in the upstream locations, together with some burnt states of low probability. On the axis at $r=0 \mathrm{~mm}$, no burnt states are observed yet and both the simulated and measured PDFs are bell-shaped with tails towards high temperatures.

At the end of this section it is instructive to investigate the PDF of temperature at locations where measured and computed mean and variance coincide, as suggested in $[55,82]$. To this end, the PDFs are compared at the same distance away from the flame root. Due to the differences between measured and computed lift-off height, the location $\mathrm{X}=50 \mathrm{~mm}$ in the experiment corresponds to $\mathrm{X}=44 \mathrm{~mm}$ in the computation. The radial profiles at these different locations for mean and variance are given in Fig. 16 for both the experiment and the simulation. Measured and computed mean and variance are almost the same if the shift in lift-off heights is considered. The agreement between measured and computed PDF depends, however, on the radial location. At a radius of $6 \mathrm{~mm}$, the computed and measured PDFs of temperature match each other well, as shown in Fig. 16. Both PDFs are bi-modal and the shape of the largest peak at about $1300 \mathrm{~K}$ is reproduced well by the computation. The smaller peak at $1870 \mathrm{~K}$ is not present in the computation, but the computed PDF increases towards high temperatures. At a radius of $7 \mathrm{~mm}$, however, a distinctive peak at $1490 \mathrm{~K}$ is visible in the computed PDF, which is not observed experimentally. This peak occurs at coflow temperature, which indicates the presence of unmixed fluid from the coflow at $\mathrm{X}=44 \mathrm{~mm}$ and $r=7 \mathrm{~mm}$. Further downstream at $\mathrm{X}=50 \mathrm{~mm}$ and $r=7 \mathrm{~mm}$, this peak is no longer present (neither in the computation nor in the experiment as shown in Fig. 15). This essentially demonstrates that comparing PDFs at different locations in space is not necessarily instructive. Even if mean and variances of experiment and computation agree well, this does not imply an agreement in terms of the PDF. This has simply to do with the fact that different PDFs may still have the same mean and variance. 

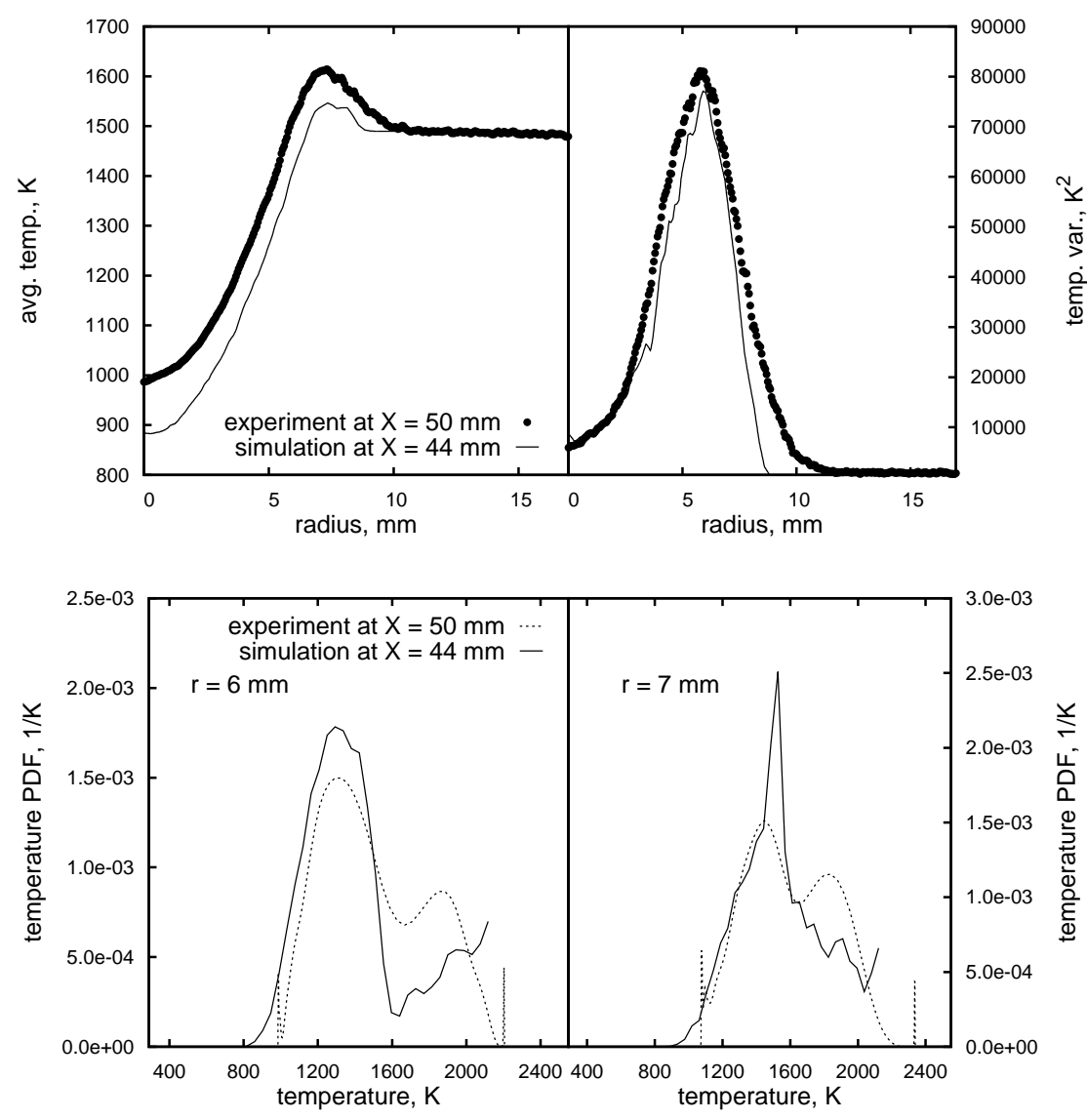

Figure 16. PDF of temperature evaluated at locations where computed mean and variance coincide.

\section{Analysis of Bi-Modality in Temperature PDF}

It is observed in Sec. 4.2 that bi-modal temperature PDFs occur in the area where the lifted flame stabilizes. In order to understand the occurrence of bi-modal temperature PDFs, it is instructive to study its transport equation, i.e. Eq. (9), under the influence of micro mixing and chemical reactions as suggested initially by Dopazo and O'Brien $[27,28]$. To this end, Eq. (9) is simplified for a system where the following assumptions apply:

- A1: The system is statistically homogeneous in terms of its thermochemical properties (the thermochemical PDFs do not vary in physical space [83]).

- A2: An irreversible, exothermal reaction occurs in this system where a component $A$ reacts to $B$ :

$$
A \stackrel{k}{\rightarrow} B
$$

The rate constant $k$ is given by the Arrhenius equation $k=$ $A_{0} \exp \left(-E_{a} /\left(R_{m} T\right)\right) . A_{0}$ is the pre-exponential factor, $E_{a}$ the activation energy, $R_{m}$ the universal gas constant, and $T$ the temperature. The molar masses of the species $A$ and $B$ are the same.

- A3: The system is closed, adiabatic, and isobaric. Under these conditions, the 
volume of the system will change due to heat release.

- A4: The specific enthalpy of the mixture is not a random variable. Due to A1 and A3 it is constant over time and in physical space.

- A5: The gradients of all thermochemical variables are zero at the boundary of the system. Hence, the gradient of any thermochemical PDF is also zero on the boundary.

- A6: The specific heat capacities of the species $A$ and $B$ are constant and equal.

- A7: The Lewis number $L e=D \rho c_{p} / \lambda$ is 1 ( $\lambda$ is the thermal conductivity and $D$ the species diffusivity). The product $\rho \cdot D$ is assumed to be constant [102].

- A8: The turbulence time scale $\tau_{t}$ and turbulent diffusivity $D_{T}$ are taken to be constant over time and independent of the spatial location.

Under the assumption A4, a random initial distribution of temperature can be induced in the system if the initial composition field is taken to be random (i.e. in each point of the system the component $A$ has reacted randomly to a certain degree to $B$ ). By utilizing the remaining assumptions, the transport equation of the temperature PDF can be simplified and solved. To this end, Eq. (9) is first integrated in physical space over the time dependent volume of the system. The resulting integral equation is simplified under the assumptions A1, A3, A5, and A8 and by applying the divergence theorem to the transport terms in physical space. Due to the convective transport in physical space, a term remains in the MDF transport equation which accounts for the effect of the volumetric expansion. In addition, the assumptions A4, A5, A6, and A7 allow us to simplify the equation further by establishing a linear relationship between the instantaneous mass fraction of $A$, i.e. $Y_{A}$, and the instantaneous temperature $T$. Note that both quantities (being random variables) vary in time as well as in physical space. Their statistics, however, vary only in time due to A1. The final evolution equation for the temperature MDF reads

$$
\frac{\partial \mathscr{F}_{T}}{\partial t}+\frac{\partial}{\partial \hat{T}}\left(\left(\omega_{\phi}(\tilde{T}(t)-\hat{T})+k\left(T_{a d}-\hat{T}\right) \mathscr{F}_{T}\right)=-\left\langle k \frac{T_{a d}-T}{T}\right\rangle \mathscr{F}_{T},\right.
$$

where $T_{a d}$ denotes the adiabatic temperature and $\omega_{\phi}=C_{\phi} /\left(2 \tau_{t}\right)$ is used here. The term on the right hand side of Eq. (23) arises due to the volumetric expansion. Equation (23) is hyperbolic, i.e. the PDF evolves from its initial state along characteristic curves. The family of characteristic curves is given for Eq. (23) by the following set of ordinary differential equations [22]

$$
\begin{aligned}
\frac{d t}{d s} & =1, \\
\frac{d \hat{T}}{d s} & =\omega_{\phi}(\tilde{T}-\hat{T})+k\left(T_{a d}-\hat{T}\right), \\
\frac{d \mathscr{F}_{T}}{d s} & =\left(\omega_{\phi}+k-\left\langle k \frac{T_{a d}-T}{T}\right\rangle\right) \mathscr{F}_{T} .
\end{aligned}
$$

$s$ is the parameter of the characteristic curves and is essentially equal to time (cf. Eq. (24)). A hyperbolic equation for the evolution of the temperature PDF in a statistically homogeneous systems is also derived by Dopazo and O'Brien in [27, 28]. Their evolution equation differs, however, from Eq. (23) for the following reasons:

(1) In equation (23) the effect of the volumetric expansion is taken into account whereas constant density is assumed by Dopazo and O'Brien [27, 28]. Since we 
consider the variability of density, we derive an evolution equation for the MDF and not for the PDF as in $[27,28]$. The volumetric expansion contributes to the inhomogeneous term of Eq. (23) and changes therefore the evolution of the MDF along the characteristic curve in Eq. (26). In general, the term is not negligible since it is directly related to heat release.

(2) Dopazo and O'Brien assume initially a large activation energy [27]. This assumption is expressed in terms of the ratio $\left\langle T_{0}\right\rangle / T_{a}$ where $\left\langle T_{0}\right\rangle$ denotes the initial expected temperature and $T_{a}=E_{a} / R_{m}$ the activation temperature. $\left\langle T_{0}\right\rangle / T_{a} \rightarrow 0$ is used in [27], which results in a linear temperature dependency of the exponent in the expression for the chemical rate constant. This assumption is not used here (later in [28] Dopazo and O'Brien also refrain from using this assumption).

(3) To include the effect of species consumption, a bi-molecular reaction is used in $[27,28]$, whereas a simple uni-molecular reaction is employed here (cf. assumption A2). The problem in the case of a bi-molecular reaction is how to statistically correlate the product of species mass fractions (which appears in the chemical source term) to temperature. In [27] the consumption of reactants is therefore neglected, based on the argument that only high activation energies are considered and that only the initial period of ignition is of interest. This assumption is problematic as it may lead to an unbounded rise in thermal energy (cf. [28]). To solve this problem, the attempt is made in [28] to include species consumption for bi-molecular reactions by using a linear relation between temperature and the product of species mass fractions. Such an assumption implies, however, that the PDF of temperature must have a lower and upper bound since the product of species mass fractions is a bounded quantity. This is not taken into account in [28] where the initial distribution of temperature is taken to be an unclipped Gaussian PDF. The effect of this inconsistency on the results is not clear.

For the following investigations it is instructive to consider the dimensionless form of Eq. (23). We introduce the following dimensionless variables

$$
\begin{aligned}
\theta & =\frac{T\left(T_{a d}-T_{u}\right)}{T_{a d}^{2}}, \\
\eta & =\omega_{\phi} t, \\
Z e & =\frac{E_{a}\left(T_{a d}-T_{u}\right)}{R_{m} T_{a d}^{2}}, \\
D a & =\frac{A_{0}}{\omega_{\phi}} e^{-\frac{E_{a}}{R_{m} T}}=D a_{\infty} e^{-\frac{Z_{e}}{\theta}}, \\
\mathscr{F}_{\theta}^{*}(\hat{\theta}) & =\frac{\mathscr{F}_{T}(\hat{T})}{\left\langle\rho_{0}\right\rangle} \frac{T_{a d}^{2}}{T_{a d}-T_{u}} .
\end{aligned}
$$

$Z e$ is the Zeldovich number [78], i.e. the dimensionless activation energy, and $D a$ the Damkoehler number which is the ratio between turbulent and chemical time scales $\left(D a_{\infty}\right.$ is the limit of $D a$ for $\left.\theta \rightarrow \infty\right)$. $\eta$ denotes the dimensionless time, $\theta$ is the dimensionless temperature, and $\hat{\theta}$ its sample space variable. For its normalization, the temperature $T_{u}$ of the unreacted component $A$ is required. $\mathscr{F}_{\theta}^{*}$ is the dimensionless mass density function of $\theta$ which is normalized by the expectation of density at the initial time $t_{0}$, i.e. $\left\langle\rho_{0}\right\rangle=\langle\rho\rangle\left(t=t_{0}\right)$. We will assume henceforth that $t_{0}=0$. The 


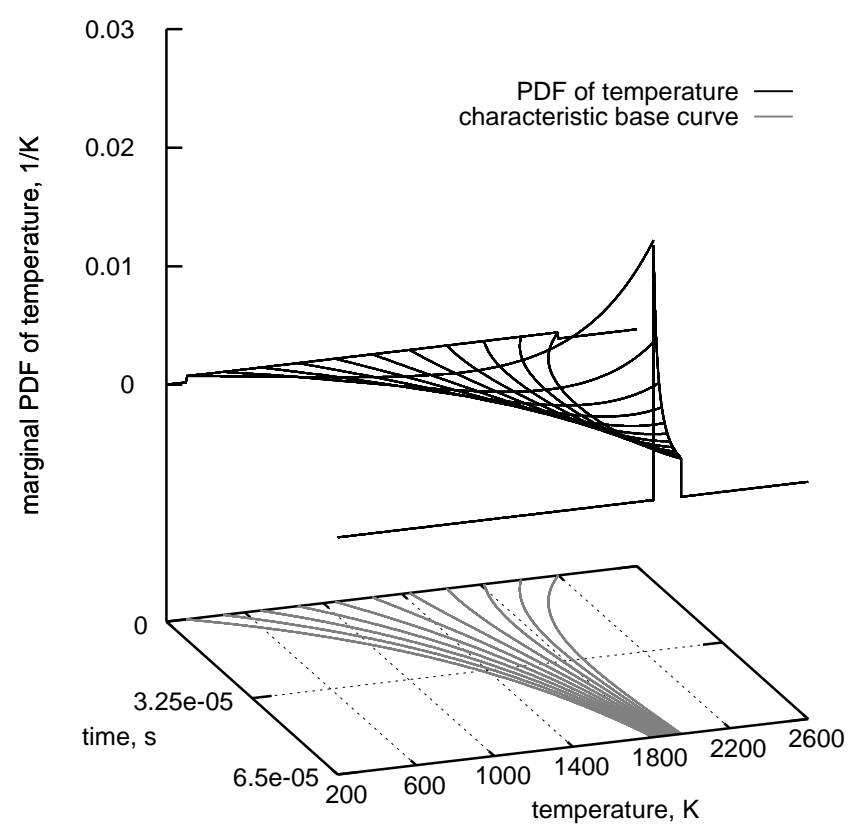

Figure 17. Marginal PDF of temperature as a function of time and temperature for $Z e=0$ and $D a=1$.

dimensionless transport equation reads

$$
\begin{aligned}
& \frac{\partial \mathscr{F}_{\theta}^{*}}{\partial \eta}+\frac{\partial}{\partial \hat{\theta}}\left(\left(\tilde{\theta}(\eta)-\hat{\theta}+D a(Z e, \hat{\theta})\left(\theta_{a d}-\hat{\theta}\right)\right) \mathscr{F}_{\theta}^{*}\right) \\
& =-\left\langle D a(Z e, \theta) \frac{\theta_{a d}-\theta}{\theta}\right\rangle \mathscr{F}_{\theta}^{*} .
\end{aligned}
$$

The evolution of the MDF depends therefore on the dimensionless numbers $Z e$ and $D a$. For $Z e=0$, i.e. for a chemical rate constant $k$ which is independent of temperature, the equations (24) to (26) can be solved analytically. The solution is given by

$$
\begin{aligned}
\hat{T}(t) & =\left(\hat{T}_{0}-\tilde{T}_{0}\right) e^{-\left(\omega_{\phi}+k\right) t}+\tilde{T}(t), \\
P_{T}(t) & =P_{T, 0} \frac{\hat{T}(t)}{\hat{T}_{0}} \frac{\tilde{T}_{0}}{\tilde{T}(t)} e^{\left(\omega_{\phi}+k\right) t}
\end{aligned}
$$

where $\tilde{T}(t)$ is given by

$$
\tilde{T}(t)=T_{a d}+\left(\tilde{T}_{0}-T_{a d}\right) e^{-k t}
$$

In these equations, $\hat{T}_{0}$ is the starting point of the characteristic base curve [22] given by Eq. (33), and $\tilde{T}_{0}$ the Favre average of temperature at time $t=0$, i.e. $\tilde{T}_{0}=\tilde{T}(t=0)$. The evolution of the PDF along the characteristic base curve depends on the initial value of the PDF at the origin of the base curve, which is defined as $P_{T, 0}=P_{T}\left(\hat{T}_{0} ; t=0\right)$. The analytical solution of the PDF as a function of temperature and time is given in Fig. 17 together with the characteristic base curves in the $t$ - $\hat{T}$-plane. The initial distribution 
of temperature is assumed to be uniform between $T_{u}=300 \mathrm{~K}$ and $T_{a d}=2200 \mathrm{~K}$ since this will clearly show the development of extrema in the PDF. $\omega_{\phi}$ and $k$ are both set to $2 \cdot 10^{4} 1 / \mathrm{s}$, i.e. the Damkoehler number is 1 . As shown in Fig. 17, the PDF becomes unimodal under these conditions. It develops an extremum at low temperatures and grows monotonically over time as its width is reduced due to micro mixing (i.e. the variance is reduced). Over time it converges asymptotically to a single Dirac pulse located at the adiabatic temperature, which corresponds to the fully reacted, perfectly mixed state of the system. It is clear from the solution given by Eqs. (33) and (34) and the illustration given in Fig. 17 that for $Z e=0$, no multi-modal solution exists for the PDF if the initial distribution is uniform. A bi-modal distribution can only be obtained for $Z e=0$ if the initial distribution $P_{T, 0}$ already possesses a maximum at high temperatures. For the general case of $Z e>0$, it is actually the non-linearity of the chemical rate constant which enables the temperature PDF to become bi-modal from an initially uniform distribution. To demonstrate this, we solve Eq. (23) numerically for this case by using a second order MacCormack method [63] in conjunction with a van Leer limiter [97]. The Zeldovich number is set here to 5.9, which corresponds to $E_{a}=30 \mathrm{kcal} /\left(\right.$ mole K) [103] if $T_{u}=300 \mathrm{~K}$ and $T_{a d}=2200 \mathrm{~K}$ are used again. These values are representative of the conditions encountered in the lifted flame. As stated before, $\omega_{\phi}$ is taken to be $2 \cdot 10^{4} 1 / \mathrm{s}$. For $A_{0}$, a value of $9 \cdot 10^{7} 1 / \mathrm{s}$ is chosen. The resulting PDF is shown in Fig. 18 at $t=10^{-5}$ s together with the range of Damkoehler numbers. It is clear that the PDF now develops a bi-modal distribution, which goes hand in hand with a considerable variation in the Damkoehler number over the temperature range where the PDF is nonzero. The minimum Damkoehler number at the lowest temperature is almost zero $\left(9.7 \cdot 10^{-13}\right)$, i.e. the chemical reaction is virtually frozen, but it increases over several orders of magnitude to a value of 2.68. Under these circumstances, fluid states at low temperatures remain almost unreacted whereas the states at high temperatures become rapidly fully reacted. Thus, the probability that both fully reacted and unreacted states occur simultaneously increases with time, resulting in a bi-modal distribution. Hence, the Zeldovich number is the important parameter here. To illustrate this, the activation energy is reduced by a factor of ten, i.e. $Z e=0.59$. At the same time the pre-exponential factor is reduced to $A_{0}=$ $1.9 \cdot 10^{5} \mathrm{1} / \mathrm{s}$. In this way, the Damkoehler number is kept the same at the adiabatic temperature. The solution is shown in Fig. 19 for $t=1 \cdot 10^{-5} \mathrm{~s}$. The PDF still develops

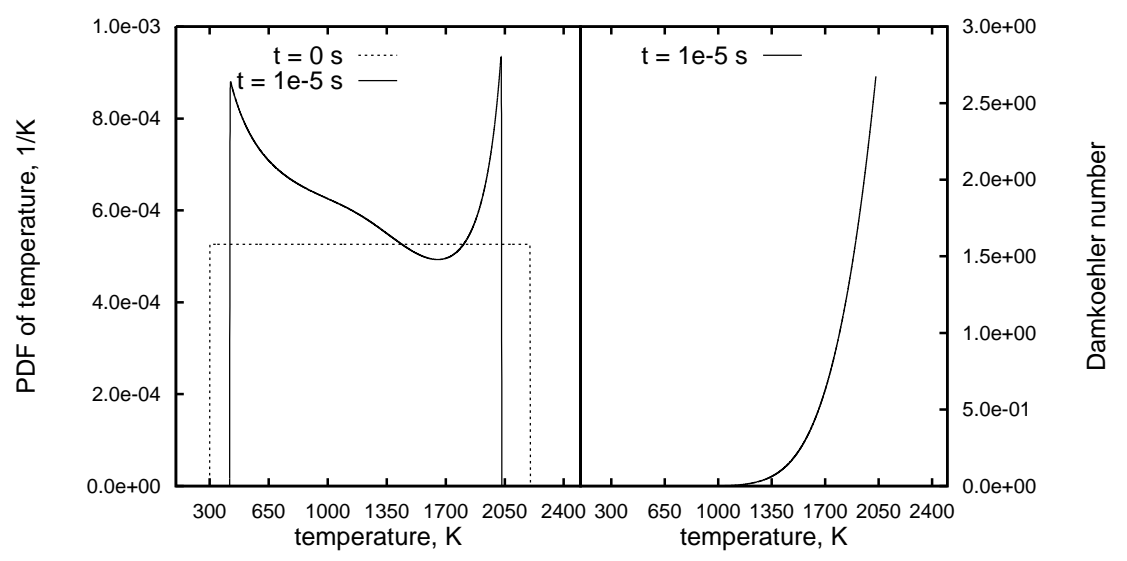

Figure 18. Temperature PDF and Damkoehler number for $Z e=5.9$. 


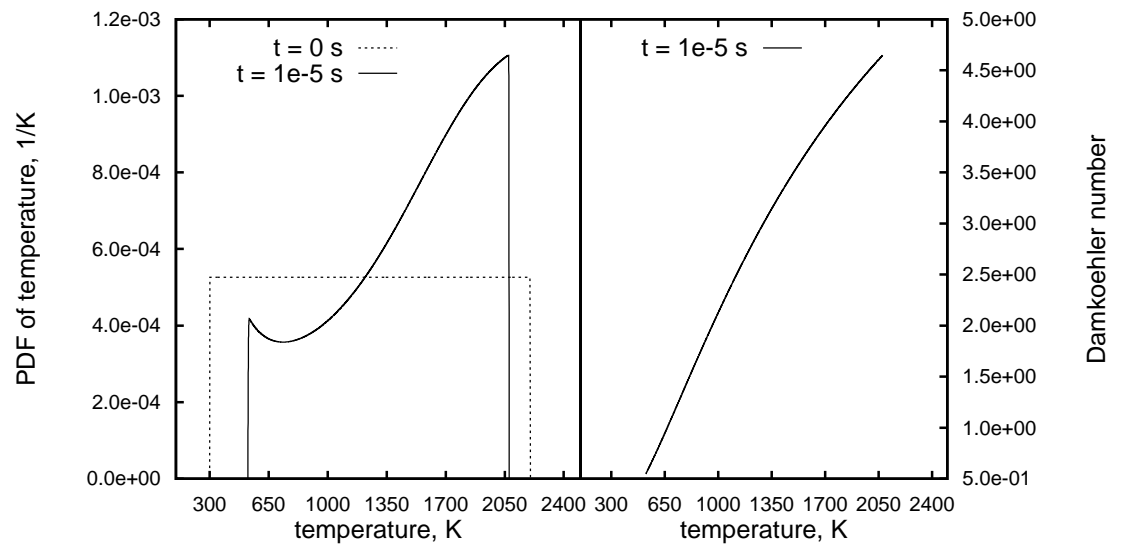

Figure 19. Temperature PDF and Damkoehler number for $Z e=0.59$.

a bi-modal shape at this point in time. However, the peak at low temperatures becomes smaller compared to the peak at high temperatures. Decreasing the Zeldovich number in the same manner even further to a value of 0.0059 results finally in a uni-modal PDF as shown in Fig. $20\left(A_{0}=9.8 \cdot 10^{4} 1 / \mathrm{s}\right.$ is used to keep the Damkoehler number constant at adiabatic temperature). The numerical solution here closely resembles the analytical solution for $Z e=0$ because the chemical rate constant hardly varies with temperature for a small activation energy. This is confirmed by the diagram of Damkoehler vs. temperature shown in Fig. 20. The Damkoehler number varies only in the range between 4.38 and 4.56 . This simplified analysis therefore suggests that the bi-modality occurs due to the temperature dependence of the chemical source term, which may be expressed in terms of the Zeldovich number. A further condition for bimodality to occur is that the Damkoehler number varies drastically in the temperature range where the $\mathrm{PDF}$ is defined. Bi-modality is observed here in situations where the Damkoehler number increases from almost zero at low temperatures to a magnitude of about one at high temperatures.

In addition to the uniform distribution, it is instructive to investigate the evolution of temperature PDFs that are initially Gaussian. The evolution of initially Gaussian

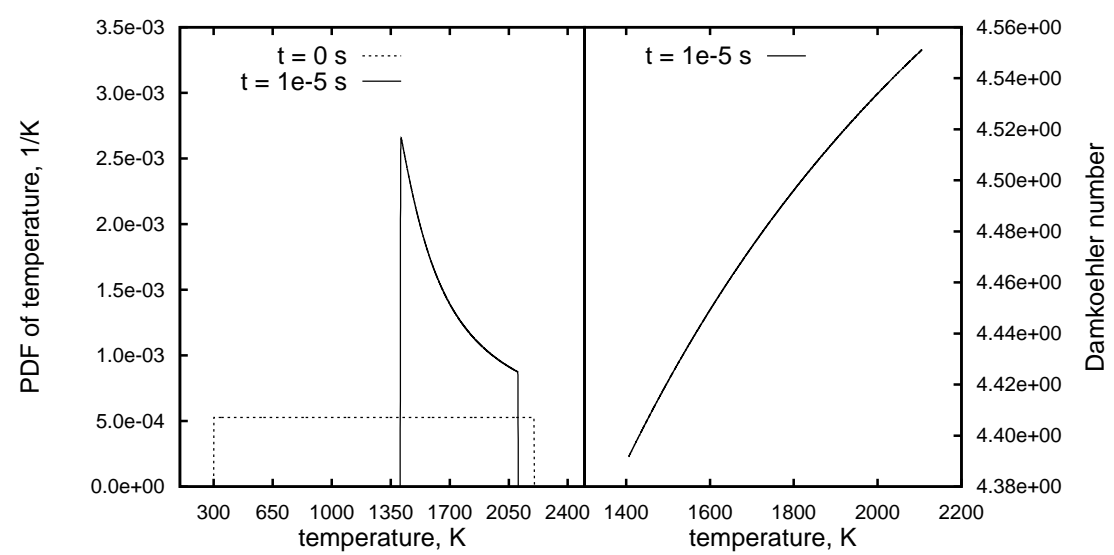

Figure 20. Temperature PDF and Damkoehler number for $Z e=0.0059$. 


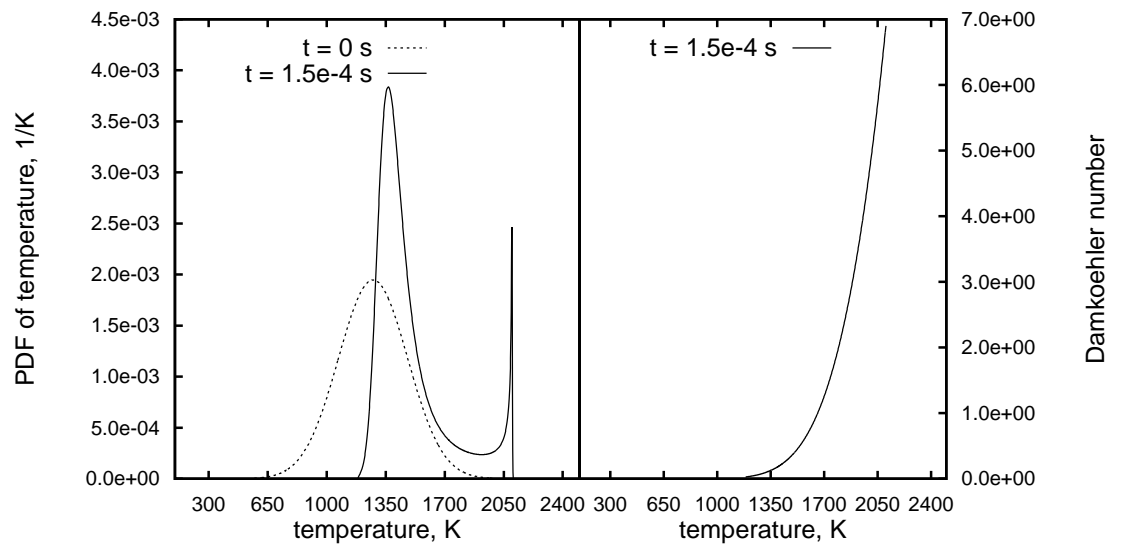

Figure 21. Temperature PDF and Damkoehler number for $Z e=5.9$ for an initially Gaussian temperature distribution. The initial root mean square is $290 \mathrm{~K}$.

PDFs during auto-ignition is already studied by Dopazo and O'Brien in [27, 28] but no bi-modality is observed by these authors. In the present work, we investigate clipped Gaussian PDFs which are scaled in order to ensure the normalization condition. In Fig. 21 the evolution of a clipped Gaussian PDF with an initial mean of $1275 \mathrm{~K}$ and a root mean square of $290 \mathrm{~K}$ is shown (the PDF is clipped initially to the range between $550 \mathrm{~K}$ and $2000 \mathrm{~K})$. As in the case of the uniform distribution, we set $Z e=5.9$, i.e. $E_{a}=30 \mathrm{kcal} /\left(\right.$ mole K), $T_{u}=300 \mathrm{~K}$, and $T_{a d}=2200 \mathrm{~K}$. We also set $A_{0}=9 \cdot 10^{7} 1 / \mathrm{s}$ but reduce $\omega_{\phi}$ to $\omega_{\phi}=1 \cdot 10^{4} 1 / \mathrm{s}$. For these conditions it is possible to obtain a bimodal PDF after $1.5 \cdot 10^{-4} \mathrm{~s}$ as shown in Fig. 21. Again we observe a considerable variation in Damkoehler number for temperatures where the PDF is nonzero. The question remains why such a bi-modality is not observed by Dopazo and O'Brien in $[27,28]$. To examine this closer, we repeat the computation for a smaller initial root mean square of $96 \mathrm{~K}$ (the PDF is clipped initially between $1035 \mathrm{~K}$ and $1515 \mathrm{~K}$ ). The resulting PDF evolution is shown in Fig. 22 after a time span of $2.5 \cdot 10^{-4} \mathrm{~s}$. Under the influence of chemistry, the PDF moves in temperature space towards higher temperatures. The width of the PDF changes over time but the PDF itself remains unimodal. A similar behavior is shown by Dopazo and O'Brien in [27]. The main reason for remaining uni-modal is that the Damkoehler number (shown in Fig. 22) does not show any major variation over the temperature range where the PDF is defined. To obtain a stronger variation in Damkoehler number it is necessary to use a larger initial variance as shown in Fig. 21. It appears, however, that Dopazo and O'Brien were not able to use large variances in their computations. They report in [27] difficulties in ensuring the normalization condition of the PDF with increasing variance, which they attribute to the fact that the characteristic base curves are displaced rapidly at high temperatures. This appears to be a plausible explanation if one takes into account that large activation energies are assumed in [27]. This linearization of the chemical rate constant under this assumption results in an unbounded growth of the chemical rate constant with increasing temperature. It is, however, more likely that the numerical scheme employed in $[27,28]$ is not well suited for solving the hyperbolic PDF equation, as difficulties to ensure the boundedness of the solution are encountered in [27]. In the present work, an explicit MacCormack scheme with a van Leer limiter is used for solving the hyperbolic MDF equation. Using this method, no boundedness violations are observed and the normalization condition is satisfied with good accuracy at all 


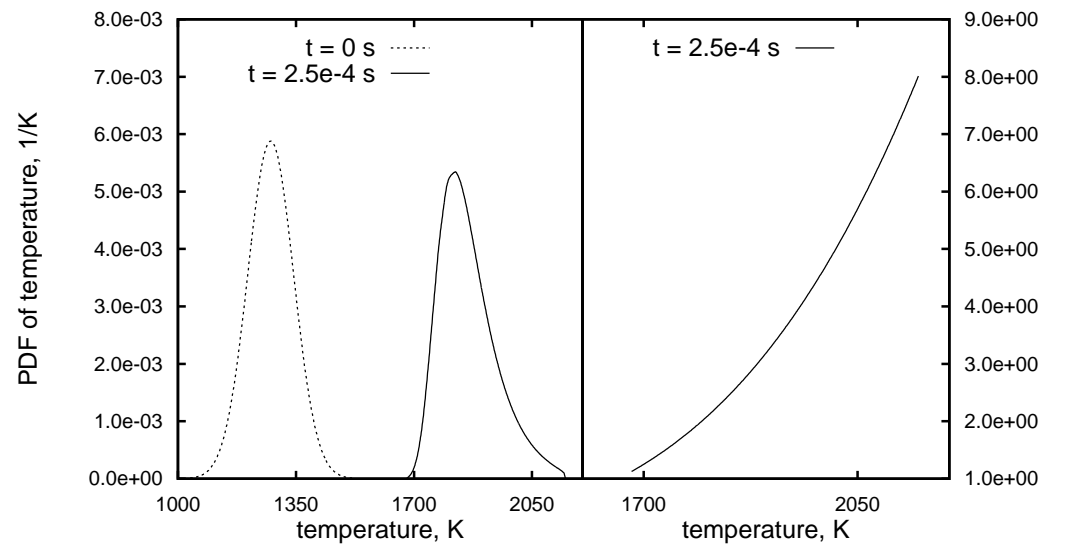

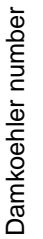

Figure 22. Temperature PDF and Damkoehler number for $Z e=5.9$ for an initially Gaussian temperature distribution. The initial root mean square is $96 \mathrm{~K}$.

times, even for large initial variances.

In order to verify if the analysis presented here applies also to conditions encountered in the flame, the Damkoehler number is investigated for each stochastic particle at locations where a bi-modal temperature PDF is observed. To this end, the thermochemical data of the stochastic particles are used to analytically compute the Jacobian matrix of the chemical source term. Its eigenvalues $\lambda_{i}$ provide a range of $N_{s}$ chemical time scales [32]

$$
\tau_{\text {chemical }, i}=\frac{1}{\left|\lambda_{i}\right|} \text {. }
$$

The largest of these time scales (i.e. the slowest chemical process) is used to determine the particle's Damkoehler number as suggested by Isaac et al. [45], i.e.

$$
\tau_{\text {chemical }}=\max _{i=1, . ., N_{s}}\left(\tau_{\text {chemical }, i}\right)
$$

As proposed in [45], principal component analysis is used in order to identify species which are chemically active. In the Jacobian matrix, only the derivatives with respect
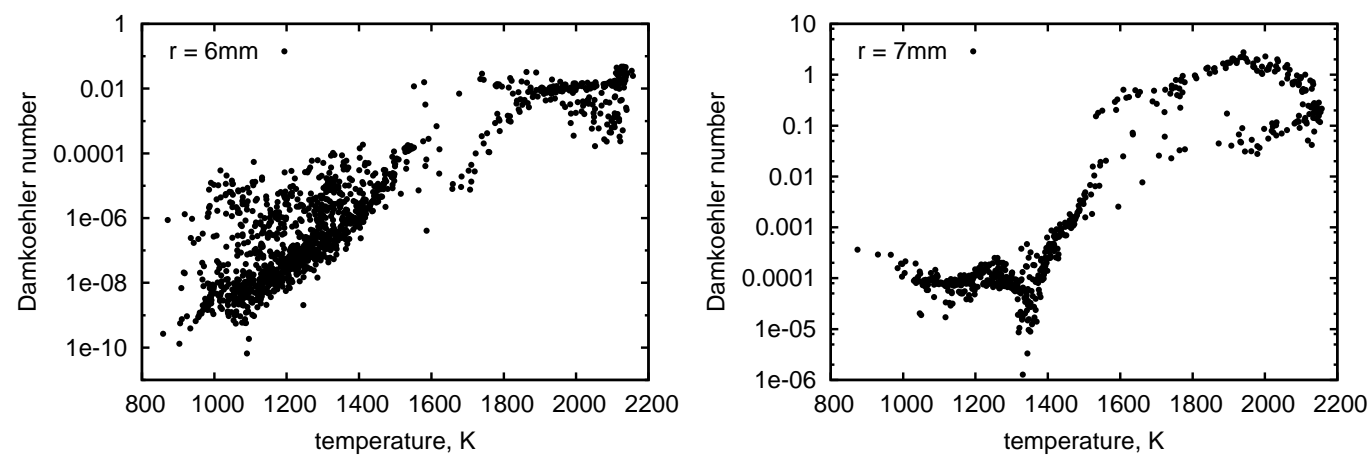

Figure 23. Damkoehler number of stochastic particles as function of particle temperature at $\mathrm{X}=50 \mathrm{~mm}$. 
to these species are considered in the estimation of $\tau_{\text {chemical }}$ [45]. Using the same procedure as in [45], the results given in Fig. 23 are obtained at the locations where bi-modal distributions occur, i.e. for $\mathrm{X}=50 \mathrm{~mm}$ at $r=7 \mathrm{~mm}$ and $r=6.5 \mathrm{~mm}$. For each particle, the Damkoehler number is plotted as a function of the particle's temperature. It turns out that a wide range of Damkoehler numbers exists at these points, as in the simplified system. For temperatures between $800 \mathrm{~K}$ and $1400 \mathrm{~K}$, the Damkoehler number drops below $10^{-3}$. Hence, compared to temperatures above $1400 \mathrm{~K}$, the chemistry is significantly slower. Under such conditions the temperature PDF can obtain a bi-modal form, as shown by this simplified analysis.

\section{Summary and Conclusions}

In the present work, a lifted methane flame in hot, vitiated air is successfully modeled by solving the transport equation of the joint thermochemical PDF by means of a Lagrangian Monte-Carlo method. A hybrid approach is employed here, where turbulence time- and length-scales are provided by solving the $k-\varepsilon$ model equations alongside the momentum equations in a finite volume solver. The computational results are compared to experimental data for $\mathrm{OH}$ concentration, temperature and mixture fraction. In general, the flame structure can be well reproduced by this method: the computed lift-off height agrees well with the lift-off height observed in the experiment. Furthermore, the average and variance of temperature are well reproduced. The comparison to average $\mathrm{OH}$ concentrations demonstrates that the location and in particular the width of the flame are captured by the model. Differences in the absolute value of $\mathrm{OH}$ concentration are mainly attributed to the slight difference between computed and measured lift-off height. In terms of average mixture fraction and mixture fraction variance, major discrepancies are found, in particular close to the fuel nozzle. These differences may be due to shortcomings in turbulence modeling. Their clarification, however, requires further experimental data, in particular for the velocity field close to the nozzle, which are not available at present. Further downstream, however, the agreement between computed and measured mixture fraction average and variance improves. Hence, the overall agreement between the available experimental data and simulation is acceptable in terms of first and second statistical moments.

To further assess the capabilities of the TPDF method in modeling auto-ignition, marginal one-point, one-time PDFs of temperature are compared to experimental data of the PDF. Such data are provided here to our knowledge for the first time in a lifted methane flame stabilized by auto-ignition. It is found experimentally for the present flame that auto-ignition results in a bi-modal distribution in temperature. Surprisingly, the highest probability of burnt states does not occur at the highest (the adiabatic) flame temperature, which would be expected if fast chemistry is to be assumed. The occurrence of bi-modal temperature PDFs is studied further in a simplified, statistically homogeneous system. It is found that the non-linear dependence of the chemical source term on temperature causes the temperature PDF to become bi-modal. It increases on the one hand the chemical reaction rate at high temperatures, and attenuates it on the other hand at low temperatures. This increases the probability of finding reacted and unreacted states at the same time, thus causing the temperature PDF to become bi-modal.

The TPDF model used in this work is capable of predicting this bi-modal PDF in the event of ignition. Studying the distribution of Damkoehler number in the TPDF simulation at locations where bi-model temperature PDFs are observed reveals similarities 
between the TPDF simulation and the simplified system. Hence, the simplified model provides a feasible explanation for the occurrence of bi-modal temperature PDFs in the flame. The chemistry in the TPDF simulation seems, however, to be faster than in the experiment, since the fully burnt states have the highest probability of occurring. This difference may be attributed to the reaction mechanism used, which might overestimate the fuel conversion rate, but the IEM mixing model might also play a role. The clarification of the effect of different reaction mechanisms and different mixing models on computational results requires further investigation.

\section{Acknowledgment}

Jeffrey Sutton, Michael Papageorge and Frederik Fuest are gratefully acknowledged for the possibility to perform high speed Rayleigh scattering measurements using the HEPBLS at the Ohio State University and their support with the data processing.

\section{References}

[1] E. Abtahizadeh, P. De Goey, and J. Van Oijen. Development of a novel flamelet-based model to include preferential diffusion effects in autoignition of $\mathrm{CH}_{4} / \mathrm{H}_{2}$ flames. Combustion and Flame, 162:4358-4369, 2015.

[2] E. Abtahizadeh, P. de Goey, and J. van Oijen. LES of Delft jet-in-hot coflow burner to investigate the effect of preferential diffusion on autoignition of $\mathrm{CH}_{4} / \mathrm{H}_{2}$ flames. Fuel, 191:36-45, 2017.

[3] C.M. Arndt, J. D. Gounder, W. Meier, and M. Aigner. Auto-ignition and flame stabilization of pulsed methane jets in a hot vitiated coflow studied with high-speed laser and imaging techniques. Applied Physics B, 108:407-417, 2012.

[4] C.M. Arndt and W. Meier. Influence of boundary conditions on the flame stabilization mechanism and on transient auto-ignition in the DLR jet-in-hot-coflow burner. Flow, Turbulence and Combustion, 2018.

[5] C.M. Arndt and W. Meier. Quantitative $\mathrm{OH}$ measurements in turbulent flames using laser-diagnostics with high spatio-temporal resolution. In Proceedings of the OSA Imaging and Applied Optics Congress - Laser Applications to Chemical, Security and Environmental Analysis, number LTu2C.3, 2018.

[6] C.M. Arndt, M.J. Papageorge, F. Fuest, J.A. Sutton, and W. Meier. Experimental investigation of the auto-ignition of a transient propane jet-in-hot-coflow. In Proceedings of the Combustion Institute, volume 37, pages 2117-2124, 2019.

[7] C.M. Arndt, M.J. Papageorge, F. Fuest, J.A. Sutton, W. Meier, and M. Aigner. The role of temperature, mixture fraction, and scalar dissipation rate on transient methane injection and auto-ignition in a jet in hot coflow burner. Combustion and Flame, 167:6071, 2016.

[8] C.M. Arndt, R. Schießel, and W. Meier. OH planar laser-induced fluorescence measurements with high spatio-temporal resolution for the study of auto-ignition. Applied Optics, 58:C14-C22, 2019.

[9] C.M. Arndt, R. Schießl, J.D. Gounder, W. Meier, and M. Aigner. Flame stabilization and auto-ignition of pulsed methane jets in a hot coflow: Influence of temperature. In Proceedings of the Combustion Institute, volume 34, pages 1483-1490, 2013.

[10] C. Bajaj, M. Ameen, and J. Abraham. Evaluation of an unsteady flamelet progress variable model for autoignition and flame lift-off in diesel jets. Combustion Science and Technology, 185:454-472, 2013.

[11] R.G. Batt. Turbulent mixing of passive and chemical reacting species in a low-speed shear layer. Journal of Fluid Mechanics, 1977. 
[12] J. Behzadi, M. Bolla, M. Talei, E.R. Hawkes, T. Lucchini, G. D'Errico, and S. Kook. Assessment of conditional moment closure for ignition in compositionally and thermally stratified mixtures. In Proceedings of the 10th Asia-Pacific Conference on Combustion, 2015.

[13] C. Bekdemir, B. Somers, and P. de Goey. DNS with detailed and tabulated chemistry of engine relevant igniting systems. Combustion and Flame, 161:210-221, 2014.

[14] C. Bekdemir, L.M.T. Somers, and L.P.H. De Goey. Modeling diesel engine combustion using pressure dependent flamelet generated manifolds. In Proceedings of the Combustion Institute, volume 33, pages 2887-2894, 2011.

[15] S. Bhattacharjee and D.C. Haworth. Simulations of transient n-heptane and n-dodecane spray flames under engine-relevant conditions using a transported PDF method. Combustion and Flame, 160:2083-2102, 2013.

[16] M. Bolla, D. Farrace, Y.M. Wright, K. Boulouchos, and E. Mastorakos. Influence of turbulence-chemistry interaction for n-heptane spray combustion under diesel engine conditions with emphasis on soot formation and oxidation. Combustion Theory and Modelling, 18:330-360, 2014.

[17] F. Bottone, A. Kronenburg, D. Gosman, and A. Marquis. The numerical simulation of diesel spray combustion with LES-CMC. Flow, Turbulence and Combustion, 89:651-673, 2012.

[18] A.J.M. Buckrell and C.B. Devaud. Investigation of mixing models and conditional moment closure applied to autoignition of hydrogen jets. Flow, Turbulence and Combustion, 90:621-644, 2013.

[19] R.R. Cao, S.B. Pope, and A.R. Masri. Turbulent lifted flames in a vitiated coflow investigated using joint PDF calculations. Combustion and Flame, 142:438-453, 2005.

[20] Z. Chen, S. Ruan, and N. Swaminathan. Simulation of turbulent lifted methane jet flames: Effects of air-dilution and transient flame propagation. Combustion and Flame, 162:703 - 716, 2015 .

[21] O. Colin and J.-B. Michel. A simplified CMC approach based on tabulated reaction rates applied to a lifted methane-air jet flame. In Proceedings of the Combustion Institute, volume 35, pages 1393-1399, 2015.

[22] R. Courant and D. Hilbert. Methods of Mathematical Physics, volume 2. Interscience Publishers, a division of John Wiley \& Sons, New York, London, 1962.

[23] G. De Paola, I.S. Kim, and E. Mastorakos. Second-order conditional moment closure simulations of autoignition of an n-heptane plume in a turbulent coflow of heated air. Flow, Turbulence and Combustion, 82:455-475, 2009.

[24] G. D'Errico, T. Lucchini, F. Contino, M. Jangi, and X.-S. Bai. Comparison of well-mixed and multiple representative interactive flamelet approaches for diesel spray combustion modelling. Combustion Theory and Modelling, 18:65-88, 2014.

[25] I. Dhuchakallaya, P. Rattanadecho, and P. Watkins. Auto-ignition and combustion of diesel spray using unsteady laminar flamelet model. Applied Thermal Engineering, 52(2):420-427, 2013.

[26] P. Domingo, L. Vervisch, and D. Veynante. Large-eddy simulation of a lifted methane jet flame in a vitiated coflow. Combustion and Flame, 152:415-432, 2008.

[27] C. Dopazo and E.E. O'Brien. An approach to the autoignition of a turbulent mixture. Acta Astronautica, 1:1239-1266, 1974.

[28] C. Dopazo and E.E. O'Brien. Statistical treatment of non-isothermal chemical reactions in turbulence. Combustion Science and Technology, 13:99-122, 1976.

[29] U. Egüz, S. Ayyapureddi, C. Bekdemir, B. Somers, and P. De Goey. Modeling fuel spray auto-ignition using the FGM approach: Effect of tabulation method. In SAE Technical Papers, 2012.

[30] A. Fiolitakis, P. R. Ess, P. Gerlinger, and M. Aigner. Development and application of a transported probability density function methond on unstructured three-dimensional grids for the prediciton of nitric oxides. Journal of Engineering for Gas Turbines and Power, 136:031506-01-031506-10, 2013. 
[31] A. Fiolitakis, P. R. Ess, P. Gerlinger, and M. Aigner. Modeling of heat transfer and differential diffusion in transported PDF methods. Combustion and Flame, 161:2107$2119,2014$.

[32] R.O. Fox. Computational Models for Turbulent Reacting Flows. Cambridge University Press, 2003.

[33] F. Fuest, M.J. Papageorge, W.R. Lempert, and J.A Sutton. Ultrahigh laser pulse energy and power generation at $10 \mathrm{kHz}$. Optics Letters, 2012.

[34] K. Gkagkas and R.P. Lindstedt. Transported PDF modelling with detailed chemistry of pre- and auto-ignition in $\mathrm{CH}_{4}$ /air mixtures. In Proceedings of the Combustion Institute, volume 31 I, pages 1559-1566, 2007.

[35] K. Gkagkas and R.P. Lindstedt. The impact of reduced chemistry on auto-ignition of $\mathrm{H}_{2}$ in turbulent flows. Combustion Theory and Modelling, 13:607-643, 2009.

[36] M.U. Göktolga, J.A. Van Oijen, and L.P.H. De Goey. Modeling MILD combustion using a novel multistage FGM method. In Proceedings of the Combustion Institute, volume 36, pages 4269-4277, 2017.

[37] D.G. Goodwin. An open-source, extensible software suite for CVD process simulation. In M. Allendorf, F. Maury, and F. Teyssandier, editors, Chemical Vapor Deposition XVI and EUROCVD 14, ECS Proceedings, volume 2003-08, pages 155-162, 2003.

[38] R. L. Gordon, A. R. Masri, S.B. Pope, and G. M. Goldin. A numerical study of autoignition in turbulent lifted flames issuing into a vitiated co-flow. Combustion Theory and Modelling, 11:351-376, 2007.

[39] C. Han, P. Zhang, T.H. Ye, and Y.L. Chen. Numerical study of methane/air jet flame in vitiated co-flow using tabulated detailed chemistry. Science China Technological Sciences, 57:1750-1760, 2014.

[40] W. Han, V. Raman, and Z. Chen. LES/PDF modeling of autoignition in a lifted turbulent flame: Analysis of flame sensitivity to differential diffusion and scalar mixing time-scale. Combustion and Flame, 171:69-86, 2016.

[41] C. Heye, V. Raman, and A.R. Masri. Influence of spray/combustion interactions on auto-ignition of methanol spray flames. In Proceedings of the Combustion Institute, volume 35, pages 1639-1648, 2015.

[42] Z. Hu, L.M.T. Somers, T. Davies, A. McDougall, and R.F. Cracknell. A study of liquid fuel injection and combustion in a constant volume vessel at diesel engine conditions. Fuel, 107:63-73, 2013.

[43] M. Ihme and Y.C. See. Prediction of autoignition in a lifted methane/air flame using an unsteady flamelet/progress variable model. Combustion and Flame, 157:1850-1862, 2010.

[44] M. Ihme and Y.C. See. LES flamelet modeling of a three-stream MILD combustor: Analysis of flame sensitivity to scalar inflow conditions. Proceedings of the Combustion Institute, 33:1309-1317, 2011.

[45] B.J. Isaac, A. Parente, C. Galletti, J.N. Thornock, P.J. Smith, and L. Tognotti. A novel methodology for chemical time scale evaluation with detailed chemical reaction kinetics. Energy \& Fuels, 27:2255-2265, 2013.

[46] M. Jangi, T. Lucchini, C. Gong, and X.-S. Bai. Effects of fuel cetane number on the structure of diesel spray combustion: An accelerated Eulerian stochastic fields method. Combustion Theory and Modelling, 19:549-567, 2015.

[47] J. Janicka, W. Kolbe, and W. Kollman. Closure of the transport equation for the probability density function of turbulent scalar fields. Journal of Non-Equilibrium Thermodynamics, 4:47-66, 1979.

[48] W.P. Jones and B.E. Launder. The prediction of laminarization with a two-equation model of turbulence. International Journal of Heat and Mass Transfer, 15:301-314, 1972.

[49] W.P. Jones and S. Navarro-Martinez. Numerical study of n-heptane auto-ignition using LES-PDF methods. Flow, Turbulence and Combustion, 83:407-423, 2009.

[50] W.P. Jones, S. Navarro-Martinez, and O. Röhl. Large eddy simulation of hydrogen auto- 
ignition with a probability density function method. In Proceedings of the Combustion Institute, volume $31 \mathrm{II}$, pages 1765-1771, 2007.

[51] T. Kamimoto and H. Kobayashi. Combustion processes in diesel engines. Progress in Energy and Combustion Science, 17:163 - 189, 1991.

[52] S.-K. Kim, Y. Yu, J. Ahn, and Y.-M. Kim. Numerical investigation of the autoignition of turbulent gaseous jets in a high-pressure environment using the multiple-RIF model. Fuel, 83:375-386, 2004.

[53] S.H. Kim, K.Y. Huh, and R.A. Fraser. Numerical prediction of the autoignition delay in a diesel-like environment by the conditional moment closure model. In SAE Technical Papers, 2000.

[54] A.Y. Klimenko and R.W. Bilger. Conditional moment closure for turbulent combustion. Progress in Energy and Combustion Science, 25:595-687, 1999.

[55] W. Kollman and J. Janicka. The probability density function of a passive scalar in turbulent shear flows. Physics of Fluids, 25, 1982.

[56] P. Kundu, T. Echekki, Y. Pei, and S. Som. An equivalent dissipation rate model for capturing history effects in non-premixed flames. Combustion and Flame, 176:202-212, 2017.

[57] B.E. Launder and B.I. Sharma. Application of the energy-dissipation model of turbulence to the calculation of flow near a spinning disk. Letters of Heat and Mass Transfer, 1:131$138,1974$.

[58] Christof Lechner and Jörg Seume. Stationäre Gasturbinen. Springer-Verlag Berlin Heidelberg, 2003.

[59] C.W. Lee and E. Mastorakos. Transported scalar PDF calculations of autoignition of a hydrogen jet in a heated turbulent co-flow. Combustion Theory and Modelling, 12:1153$1178,2008$.

[60] H. Lehtiniemi, F. Mauss, M. Balthasar, and I. Magnusson. Modeling diesel spray ignition using detailed chemistry with a progress variable approach. Combustion Science and Technology, 178:1977-1997, 2006.

[61] J. Lim, Y. Kim, S. Lee, J. Chung, W. Kang, and K. Min. 3-d simulation of the combustion process for di-methyl ether-fueled diesel engine. Journal of Mechanical Science and Technology, 24:2597-2604, 2010.

[62] J. Löwe, A. Probst, T. Knopp, and R. Kessler. Low-dissipation low-dispersion secondorder scheme for unstructured finite volume flow solvers. AIAA Journal, 54:2961-2971, 2016.

[63] R.W. MacCormack. The effect of viscosity in hypervelocity impact cratering. Number AIAA 69-354, 1969.

[64] A.R. Masri, R. Cao, S.B. Pope, and G.M. Goldin. PDF calculations of turbulent lifted flames of $\mathrm{H}_{2} / \mathrm{N}_{2}$ fuel issuing into a vitiated co-flow. Combustion Theory and Modelling, $8: 1-22,2004$.

[65] E. Mastorakos. Ignition of turbulent non-premixed flames. Progress in Energy and Combustion Science, 35:57-97, 2009.

[66] J.-B. Michel and O. Colin. A tabulated diffusion flame model applied to diesel engine simulations. International Journal of Engine Research, 15:346-369, 2014.

[67] S.M. Mir Najafizadeh, M.T. Sadeghi, R. Sotudeh-Gharebagh, and D.J.E.M. Roekaerts. Chemical structure of autoignition in a turbulent lifted $\mathrm{H}_{2} / \mathrm{N}_{2}$ jet flame issuing into a vitiated coflow. Combustion and Flame, 160:2928-2940, 2013.

[68] H. Möbus, P. Gerlinger, and D. Brüggemann. Comparison of Eulerian and Lagrangian monte carlo PDF methods for turbulent diffusion flames. Combustion and Flame, 124:519-534, 2001.

[69] M.G. Mungal and P.E. Dimotakis. Mixing and combustion with low heat release in a turbulent shear layer. Journal of Fluid Mechanics, 148:349-382, 1984.

[70] B. Naud, R. Novella, J.M. Pastor, and J.F. Winklinger. RANS modelling of a lifted $\mathrm{H}_{2} / \mathrm{N}_{2}$ flame using an unsteady flamelet progress variable approach with presumed PDF. Combustion and Flame, 162:893-906, 2015. 
[71] S. Navarro-Martinez and A. Kronenburg. Analysis of stabilization mechanisms in lifted flames. AIP Conference Proceedings, 1190:13-38, 2009.

[72] S. Navarro-Martinez and A. Kronenburg. Flame stabilization mechanisms in lifted flames. Flow, Turbulence and Combustion, 87:377-406, 2011.

[73] S. Navarro-Martinez and S. Rigopoulos. Large eddy simulation of a turbulent lifted flame using conditional moment closure and rate-controlled constrained equilibrium. Flow, Turbulence and Combustion, 87:407-423, 2011.

[74] P. Pal, S. Keum, and H.G. Im. Assessment of flamelet versus multi-zone combustion modeling approaches for stratified-charge compression ignition engines. International Journal of Engine Research, 17:280-290, 2016.

[75] M.J. Papageorge, C. Arndt, F. Fuest, W. Meier, and J.A. Sutton. High-speed mixture fraction and temperature imaging of pulsed, turbulent fuel jets auto-igniting in hightemperature, vitiated co-flows. Experiments in Fluids, 55:1763, 2014.

[76] M.J. Papageorge, T.A. McManus, F. Fuest, and J.A. Sutton. Recent advances in highspeed planar rayleigh scattering in turbulent jets and flames: increased record lengths, acquisition rates, and image quality. Applied Physics B, 2014.

[77] S.S. Patwardhan, De Santanu, K.N. Lakshmisha, and B.N. Raghunandan. CMC simulations of lifted turbulent jet flame in a vitiated coflow. In Proceedings of the Combustion Institute, volume 32, pages 1705-1712, 2009.

[78] Norbert Peters. Turbulent Combustion. Cambridge monographs on mechanics, 2006.

[79] E.L. Petersen, M. H. Joel, D.S. Schuyler, J. de Vries, A. R. Amadio, and M.W. Crofton. Ignition of lean methane-based fuel blends at gas-turbine pressures. Journal of Engineering for Gas Turbines and Power, 129:937-944, 2007.

[80] S. B. Pope. The probability approach to the modelling of turbulent reacting flows. Combustion and Flame, 27:299-312, 1976.

[81] S. B. Pope. PDF methods for turbulent reactive flows. Progress in Energy and Combustion Science, 11:119-192, 1985.

[82] S.B. Pope. A Monte Carlo Method for the PDF Equations of Turbulent Reactive Flow. Combustion Science and Technology, 25:159-174, 1981.

[83] Stephen B. Pope. Turbulent Flows. Cambridge University Press, 2009.

[84] J. Prause, C.M. Arndt, B. Noll, and M. Aigner. Large-eddy-simulation of auto-ignition in a turbulent jet in hot coflow. In Thirteenth international workshop on measurement 83 computation of turbulent flames, 2016.

[85] G. Reichling, B. Noll, and M. Aigner. Development of a projection-based method for the numerical calculation of compressible reactive flows. In Proceedings of the 51st AIAA Aerospace Sciences Meeting including the New Horizons Forum and Aerospace Exposition, number AIAA 2013-1003, 2013.

[86] V. Sabel'nikov, M. Gorokhovski, and N. Baricault. The extended IEM mixing model in the framework of the composition PDF approach: Applications to diesel spray combustion. Combustion Theory and Modelling, 10:155-169, 2006.

[87] Gregory P. Smith, David M. Golden, Michael Frenklach, Nigel W. Moriarty, Boris Eiteneer, Mikhail Goldenberg, C. Thomas Bowman, Ronald K. Hanson, Soonho Song, Jr. William C. Gardiner, Vitali V. Lissianski, and Zhiwei Qin. Gri 3.0 mechanism. Online; accessed September 20th, 2008. http://www.me.berkeley.edu/gri_mech/, 1999.

[88] I. Stankovic, E. Mastorakos, and B. Merci. LES-CMC simulations of different autoignition regimes of hydrogen in a hot turbulent air co-flow. Flow, Turbulence and Combustion, 90:583-604, 2013.

[89] I. Stankovic and B. Merci. Analysis of auto-ignition of heated hydrogen-air mixtures with different detailed reaction mechanisms. Combustion Theory and Modelling, 15:409-436, 2011.

[90] I. Stankovic and B. Merci. LES-CMC simulations of a turbulent lifted hydrogen flame in vitiated co-flow. Thermal Science, 17:763-772, 2013.

[91] I. Stankovic, A. Triantafyllidis, E. Mastorakos, C. Lacor, and B. Merci. Simulation of hydrogen auto-ignition in a turbulent co-flow of heated air with LES and CMC approach. 
Flow, Turbulence and Combustion, 86:689-710, 2011.

[92] S. Subramaniam and S.B. Pope. A mixing model for turbulent flows based on euclidian minimum spanning trees. Combustion and Flame, 115:487-514, 1998.

[93] B. Talbot, N. Mazellier, B. Renou, L. Danaila, and M.A. Boukhalfa. Time-resolved velocity and concentration measurements in variable-viscosity turbulent jet flow. Experiments in Fluids, 47:769-787, 2009.

[94] J. Tillou, J.-B. Michel, C. Angelberger, and D. Veynante. Assessing LES models based on tabulated chemistry for the simulation of diesel spray combustion. Combustion and Flame, 161:525-540, 2014.

[95] A. Tyliszczak. Assessment of implementation variants of conditional scalar dissipation rate in LES-CMC simulation of auto-ignition of hydrogen jet. Archives of Mechanics, 65:97-129, 2013.

[96] A. Tyliszczak. LES-CMC study of an excited hydrogen flame. Combustion and Flame, 162:3864-3883, 2015.

[97] B. van Leer. Towards the ultimate conservative difference scheme, V: A second-order sequel to Godunov's method. Journal of Computational Physics, 32:101-136, 1979.

[98] K. S. Venkataramani, N. K. Tutu, and R. Chevray. Probability distributions in a round heated jet. Physics of Fluids, 18:1413-1420, 1975.

[99] H.K. Versteeg and W. Malalasekera. An Introduction to computational fluid dynamics: The finite volume method. Pearson Prentice Halls, 1995.

[100] J. Villermaux and J.C. Devillon. Representation de la coalescence et de la redispersion des domaines de segregation dans un fluide par un modele d' interaction phenomenologique. In Second International Symposium on Chemical Reaction Engineering, pages 1-13, New York, 1972. Elsevier.

[101] H. Wang and S. Pope. Lagrangian investigation of local extinction, re-ignition and auto-ignition in turbulent flames. Combustion Theory and Modelling, 12:857-882, 2008.

[102] J. Warnatz, U. Maas, and R.W. Dibble. Combustion: Physical and Chemical Fundamentals, Modeling and Simulation, Experiments, Pollutant Formatinon. Springer, 1995.

[103] C.K. Westbrook and F.L. Dryer. Simplified reaction mechanisms for the oxidation of hydrocarbon fuels in flames. Combustion Science and Technology, 27:31-43, 1981.

[104] Y.M. Wright, O.-N. Margari, K. Boulouchos, G. De Paola, and E. Mastorakos. Experiments and simulations of n-heptane spray auto-ignition in a closed combustion chamber at diesel engine conditions. Flow, Turbulence and Combustion, 84:49-78, 2010.

[105] Y. Yu, S.-K. Kim, and Y.-M. Kim. Numerical modeling for auto-ignition and combustion processes of fuel sprays in high-pressure environment. Combustion Science and Technology, 168:85-112, 2001.

[106] H. Zhou, S. Li, Z. Ren, and D.H. Rowinski. Investigation of mixing model performance in transported PDF calculations of turbulent lean premixed jet flames through lagrangian statistics and sensitivity analysis. Combustion and Flame, 181:136-148, 2017. 